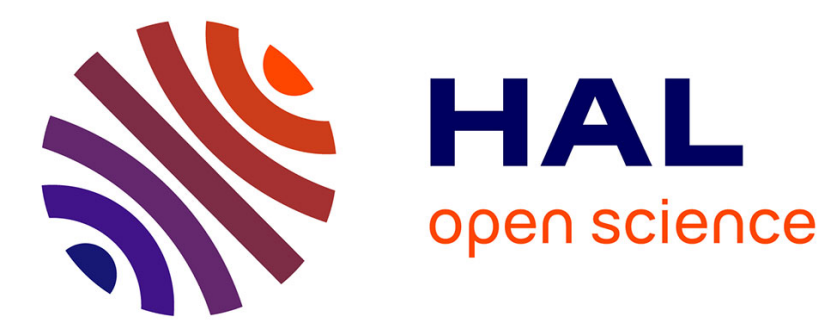

\title{
Local Turn-Boundedness, a curvature control for continuous curves with application to digitization
}

Etienne Le Quentrec, Loïc Mazo, Étienne Baudrier, Mohamed Tajine

\section{To cite this version:}

Etienne Le Quentrec, Loïc Mazo, Étienne Baudrier, Mohamed Tajine. Local Turn-Boundedness, a curvature control for continuous curves with application to digitization. Journal of Mathematical Imaging and Vision, 2020, 62 (5), pp.673-692. 10.1007/s10851-020-00952-x . hal-02891118

\section{HAL Id: hal-02891118 https://hal.science/hal-02891118}

Submitted on 6 Jul 2020

HAL is a multi-disciplinary open access archive for the deposit and dissemination of scientific research documents, whether they are published or not. The documents may come from teaching and research institutions in France or abroad, or from public or private research centers.
L'archive ouverte pluridisciplinaire $\mathbf{H A L}$, est destinée au dépôt et à la diffusion de documents scientifiques de niveau recherche, publiés ou non, émanant des établissements d'enseignement et de recherche français ou étrangers, des laboratoires publics ou privés. 


\title{
Local Turn-Boundedness, a curvature control for continuous curves with application to digitization
}

\author{
Étienne Le Quentrec, Loïc Mazo, Étienne Baudrier and Mohamed Tajine
}

March 13, 2020

\begin{abstract}
This article focuses on the classical problem of the control of information loss during the digitization step. The properties proposed in the literature rely on smoothness hypotheses that are not satisfied by the curves including angular points. The notion of turn introduced by Milnor in the article On the Total Curvature of Knots generalizes the notion of integral curvature to continuous curves. Thanks to the turn, we are able to define the local turn-boundedness. This promising property of curves does not require smoothness hypotheses and shares several properties with the $\operatorname{par}(r)$-regularity, in particular well-composed digitizations. Besides, the local turn-boundedness enables to constrain spatially the continuous curve as a function of its digitization.
\end{abstract}

\section{Introduction}

The loss of information caused by a digitization process is inevitable. Therefore a fundamental point concerns the control of this information loss. This is the starting point for our study. More precisely, we want to determine conditions under which the discretization of a shape preserves - in a sense to be specified - some of the geometric and topological properties of the original continuous shape. Then we focus on a geometric criterion, the control of the Hausdorff distance between a shape and its digitization, and a topological criterion, the preservation of the manifoldness of a shape.

Several hypotheses on the shape have been proposed in the literature to obtain such faithful digitizations. These hypotheses are detailed hereafter and compared in Table 1 for a set of properties. In the rest of the paper, $S$ stands for a shape of the Euclidean plane whose border $\mathcal{C}$ is a Jordan curve.

One of the most used hypotheses, called par(r)-regularity, was independently introduced in 1982 by Pavlidis in [13] and in 1984 by Serra in [14] in order to study the preservation of the topology by the Gauss digitization. It demands that any point $c \in \mathcal{C}$ has an interior osculating disk entirely included 
in the interior of $\mathcal{C}$ except for the point $c$ and an exterior osculating disk entirely included in the exterior of $\mathcal{C}$ except for the point $c$ (see Definition 10). It has been used to prove some topology preservation properties $[5,8,13]$. The $\operatorname{par}(r)$-regularity encompasses two ideas: the border of the shape has a curvature bounded from above and the shape has a positive minimal thickness. But $\operatorname{par}(r)$-regularity fails to include non-regular shapes having corners as polygons (see Proposition 10). That is why there exists in the literature many notions generalizing $\operatorname{par}(r)$-regularity. For instance, Stelldinger et al. suggest in $[15,16]$ a regularization to transform some class of shapes (half-regular shapes) having spikes into $\operatorname{par}(r)$-regular shapes. The half-regularity is a more general version than par-regularity. Indeed, it only demands that each point on the border of the shape has one of the two osculating disks modulo the exclusion of a kind of defects in the regularization process. In [10], Meine et al. introduced a generalization of half $(r)$-regularity: the $r$-stability. A shape is $r$-stable if its boundary can be dilated with a closed disk of radius $s$ without changing its homotopy type for any $s \leq r$. Sadly, these two latest notions give no guarantee about the well-composedness of the digitization but only about the homotopy equivalence between the object and its reconstruction and do not provide a control of the geometry. Indeed, they allow the continuous shape to be arbitrarily far from its digitization. More controlled generalizations of $\operatorname{par}(r)$-regularity under digitization have been developed. For instance, Ngo et al. [12] define the notion of quasi(r)-regularity allowing irregularities of the border of the shape $S$ to lie in a margin of magnitude $(\sqrt{2}-1) r$ around the erosion of $S$ by a centered ball of radius $r$. The quasi-regularity has been introduced in order to guarantee the preservation under rigid transformation of the well-composedness. But the definition of quasi $(r)$-regularity uses both local and global properties as connectedness. Moreover, given explicitly a shape $S$, it can be hard to determine whether it is quasi $(r)$-regular. In [10], in addition to $r$-stability, Meine et al. also defined the $(\theta, d)$-spikesas arcs delimited by two points $x_{1}$ and $x_{2}$ at distance at most $d$ from each other, such that there exists a point $y$ in this arc forming an angle $\widehat{x_{1} y x_{2}}$ strictly less than $\theta$. Alone, the notion of curve without $(\theta, d)$-spike can only give a bound on the distance between a shape $S$ and its digitization. The classical notion of reach [4] which is the minimal distance between the boundary of a shape and its medial axis also makes it possible to control the thickness and the curvature. Indeed, it was proven in [7], that par-regularity amounts to asking a positive reach. But having positive reach requires continuous differentiability of the boundary. In [3], Chazal et al. defined the $\mu$-reach that just requires that all the projections on the curve of a medial axis point close to the curve are seen under some tight angle. Nevertheless, the $\mu$-reach seems hard to compute and does not guarantee the well-composedness of the digitization but only the homotopy equivalence.

In this article, we introduce a notion that requires the shape to be thick enough and not to have small artifacts in comparison with the grid step. In other words, the border of $S$ should be locally flat. We propose a new wide class of Jordan curves whose interior fulfill the previous requirements. We call them locally turn-bounded curves. By wide class, we mean a class that encompasses 
both regular curves and polygons. The thickness part of the definition is based on the distortion thickness of Kusner and Sullivan [6]. Their idea is to consider the minimal distance between two points of the curve sufficiently far from each other for the geodesic distance. The locally flatness part of the definition of the locally turn-boundedness relies on the notion of turn adapted to both regular curves and polygons. The turn was firstly introduced by Milnor [11] to study the geometry of knots. We get the local turn-boundedness by replacing the geodesic distance with the total curvature in the definition of the distortion thickness. Indeed, local turn-boundedness allows us a more acute control on the curve. For instance, instead of bounding one arc delimited by two fixed points in an ellipse

whose foci are the two points, we bound the arc in a disk whose diameter is the segment delimited by the two points.

This article is an extended version of the conference article [9]. The additions are the following: a stronger result about well-composedness (Proposition 9), the digitization of a locally turn-bounded curve is 4-connected (Corollary 5) and a proof that a $\operatorname{par}(r)$-regular curve is locally turn-bounded (Section 5). In order to prove these results, technical lemmas, propositions and definitions have been introduced.

The article is organized as follows. First, we recall the main properties and definitions about the notion of turn (Section 2). Then, we present the class of locally turn-bounded curves and we give their basic properties (Section 3). Section 4 is devoted to the proof of our first main result, Theorem 1: the digitization of a shape bounded by a locally turn-bounded curve is well-composed and 4-connected under a "compatibility hypothesis" related to the grid step. In Section 5, we prove our second main result, Theorem 2: local turn-boundedness is a generalization of par-regularity (and thus, of having positive reach).

\section{Turn of a simple curve}

Although the notion was introduced by Milnor in [11], the definitions and properties given in this section come from the book of Alexandrov and Reshetnyak [1]. As presented in Proposition 2, the turn extends to continuous curves the notion of integral curvature already defined for regular curves.

Terminology and notations In this paragraph, some necessary notions on oriented curves are recalled.

- Let $c \in \mathbb{R}^{2}$ and $r \geq 0$. We denote by $B(c, r)$ the open disk of center $c$ and radius $r$ and by $\bar{B}(c, r)$ the close disk of center $c$ and radius $r$.

- A parametrized curve is a continuous application $\gamma$ from an interval $\left[t_{0}, t_{1}\right]$ of $\mathbb{R}, t_{0}<t_{1}$, to $\mathbb{R}^{2}$. It is simple if it is injective on $\left[t_{0}, t_{1}\right)$ and closed if $\gamma\left(t_{0}\right)=\gamma\left(t_{1}\right)$. A (geometric) curve is the image of a parametrized curve. A Jordan curve is a simple closed curve. 


\begin{tabular}{|c|c|c|c|}
\hline Notion & $\begin{array}{l}\text { digitization well- } \\
\text { composed }\end{array}$ & $\begin{array}{ll}\text { digitization } \\
\text { connected }\end{array}$ & $\begin{array}{l}\text { distance to the dig- } \\
\text { itization }\end{array}$ \\
\hline \multirow{5}{*}{$\begin{array}{c}\text { par-regularity } \\
\text { half-regularity } \\
r \text {-stability } \\
\mu \text {-reach } \\
\text { quasi-regularity } \\
\text { local turn- } \\
\text { boundedness }\end{array}$} & Theorem 7.1 [13] & Theorem 7.1 [13] & Theorem 1 [7] \\
\hline & no & & \\
\hline & & & \\
\hline & Pronocition 2 [12] & Drongition 2 [12] & \\
\hline & Proposition 9 & Proposition 5 & Proposition 6 \\
\hline Notion & $\begin{array}{l}\text { same homotopy } \\
\text { tree with a recon- } \\
\text { struction }\end{array}$ & $\begin{array}{l}\text { homotopy equiv- } \\
\text { alence with a } \\
\text { reconstruction }\end{array}$ & $\begin{array}{l}\text { homotopy } \text { equiv- } \\
\text { alence with the } \\
\text { dilation of an } \\
\text { approximation }\end{array}$ \\
\hline $\begin{array}{c}\text { half-regularity } \\
r \text {-stability } \\
\mu \text {-reach }\end{array}$ & Theorem 14 [16] & Theorem 11 [10] & Theorem $4.6[3]$ \\
\hline
\end{tabular}

Table 1: This table sums up the notions mentioned in the introduction. Notice that in the fourth and fifth column the reconstruction depends on each notion.

- For a simple parametrized curve $\gamma$, an order is defined on the points of the associated curve $\mathcal{C}$ by:

$$
\gamma(\alpha) \leq_{\gamma} \gamma(\beta) \Leftrightarrow \alpha \leq \beta
$$

and $\leq_{\gamma}$ is denoted by $\leq$ if there is no ambiguity. A simple curve $\gamma$ with such an order is called oriented curve.

- A polygonal line with vertices $x_{0}, \ldots, x_{N}$ is denoted by $\left[x_{0}, x_{1} \ldots, x_{N}\right]$ (if $x_{N}=x_{0}$, the polygonal line is a polygon).

A polygonal line $L$ is inscribed into an oriented curve $\mathcal{C}$ if the vertices of the polygonal line $L$ form an increasing sequence for the order relationship defined by some simple parametrization of $\mathcal{C}$. For a Jordan curve, a polygonal line $L$ is inscribed if its first and its last vertex are equal and all its vertices but the last form an increasing sequence for the order relationship defined by some simple parametrization of $\mathcal{C}$.

- Let $N$ be a positive integer and $x_{0}, x_{1}, \ldots, x_{N}$ points of $\mathbb{R}^{2}$. The polygonal line PL $=\left[x_{0}, x_{1}, \ldots, x_{N}\right]$ can be considered as the image of the parametrized curve $p l:[0, N] \mapsto \mathbb{R}^{2}$ such that $p l(t)=x_{\lfloor t\rfloor}(t-\lfloor t\rfloor)+(1-$ $t+\lfloor t\rfloor) x_{\lfloor t\rfloor+1}$ where for $r \in \mathbb{R},\lfloor r\rfloor$ in the integer part of the real $r$. In other words, for any integer $i$ between 0 and $N$, if $t \in[i, i+1)$, then $p l(t)=(t-i) x_{i}+(1-t+i) x_{i+1}$, and thus $p l([i, i+1])$ is the segment $\left[x_{i}, x_{i+1}\right]$ of $\mathbb{R}^{2}$. A polygonal line is simple if it is simple for the previous parametrization and thus a simple polygon is a Jordan curve.

- Given a curve $\mathcal{C}$ and two points $a, b$ on $\mathcal{C}(a \neq b)$, we write $\mathcal{C}_{a}^{b}$ for the arc ending at $a$ and $b$ if $\mathcal{C}$ is not closed. If $\mathcal{C}$ is closed, $\mathcal{C}_{a}^{b}$ and $\mathcal{C}_{b}^{a}$ stand for 
the two arcs of $\mathcal{C}$ delimited by $a$ and $b$. Since these two arcs are hard to distinguish formally, the latter notation is defined up to permutation of the two arcs.

- The angle between two vectors $\mathbf{u}$ and $\mathbf{v}$ is denoted by $(\mathbf{u}, \mathbf{v})((\mathbf{u}, \mathbf{v}) \in$ $\mathbb{R} / 2 \pi \mathbb{Z})$. The geometric angle between two vectors $\mathbf{u}$ and $\mathbf{v}$, denoted by $\angle(\mathbf{u}, \mathbf{v})$, or two directed straight lines oriented by $\mathbf{u}$ and $\mathbf{v}$, is the absolute value of the reference angle taken in $(-\pi, \pi]$ between the two vectors. Thus, $\angle(\mathbf{u}, \mathbf{v}) \in[0, \pi]$. Given three points $x, y, z$, we also write $\widehat{x y z}$ for the geometric angle between the vectors $x-y$ and $z-y$.

Remark 1. Every geometric curve is a compact set. Hence the straight lines are not geometric curves.

Definition 1 (Turn). defined by:

- The turn $\kappa(L)$ of a polygonal line $L=\left[x_{i}\right]_{i=0}^{N}$ is

$$
\kappa(L):=\sum_{i=1}^{N-1} \angle\left(x_{i}-x_{i-1}, x_{i+1}-x_{i}\right) .
$$

- The turn $\kappa(P)$ of a polygon $P=\left[x_{i}\right]_{i=0}^{N}$ (where $x_{N}=x_{0}$ and $\left.x_{N+1}=x_{1}\right)$ is defined by:

$$
\kappa(P):=\sum_{i=1}^{N} \angle\left(x_{i}-x_{i-1}, x_{i+1}-x_{i}\right) .
$$

- The turn $\kappa(\mathcal{C})$ of a simple curve $\mathcal{C}$ (respectively of a Jordan curve) is the upper bound of the turn of its inscribed polygonal lines (respectively of its inscribed polygons).

Since the turn of a polygon equals the upper bound of the turn of the polygons inscribed in it [1, Corollary p. 119], the turn of the polygon seen as a closed curve is equal to the turn of the polygon. Hence, the turn is well defined.

It should be noticed that the turn does not depend on the orientation of the curve. Indeed, it is well-known that $(\mathbf{u}, \mathbf{v})=-(-\mathbf{v},-\mathbf{u})$, then $\angle(\mathbf{u}, \mathbf{v})=$ $\angle(-\mathbf{u},-\mathbf{v})$. Thus $\kappa\left(\left[x_{i}\right]_{i=0}^{N}\right)=\kappa\left(\left[x_{i}\right]_{i=N}^{0}\right)$.

Remark 2. The turn is stable under homothetic maps. Indeed, obviously, the turn is invariant by any conformal map, in particular by the homotheties.

Like the length of a curve, the turn can be calculated thanks to multiscale samplings. This is the object of Property 1 where we denote by $\mathcal{L}(\mathcal{C})$ the length of the curve $\mathcal{C}$.

Property 1 (Convergence of the length and turn of a sequence of polygonal lines [1], p. 23, 30, 121, 122). Let $\mathcal{C}$ be a simple curve and $\left(L_{m}\right)_{m \in \mathbb{N}}$ a sequence of polygonal lines inscribed in $\mathcal{C}$ and with same endpoints as $\mathcal{C}$. If $\lim _{m \rightarrow+\infty} \lambda_{m}=$ 0 , where $\lambda_{m}$ is the maximum length of a side of the polygonal line $L_{m}$, then

$$
\lim _{m \rightarrow+\infty} \mathcal{L}\left(L_{m}\right)=\mathcal{L}(\mathcal{C})
$$




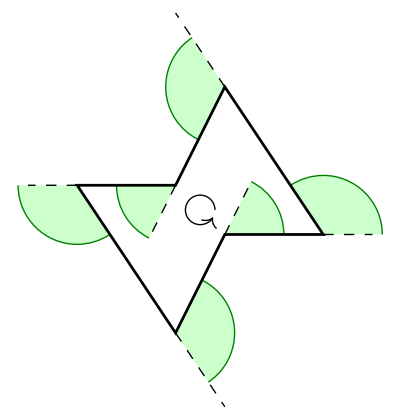

Figure 1: The turn of the polygon is the sum of the green angles.

and

$$
\lim _{m \rightarrow+\infty} \kappa\left(L_{m}\right)=\kappa(\mathcal{C}) .
$$

Moreover, if $\kappa(\mathcal{C})$ is finite, then $\mathcal{L}(\mathcal{C})$ is also finite (i. e. $\mathcal{C}$ is rectifiable).

In Property 1, if we assume that the sequence $\left(L_{m}\right)$ is increasing $\left(L_{m}\right.$ is inscribed in $\left.L_{m+1}\right)$, then the sequences $\left(\mathcal{L}\left(L_{m}\right)\right)$ and $\left(\kappa\left(L_{m}\right)\right)$ are both increasing [1, Lemma 5.1.1].

Property 2 (Turn for regular curves [1], p. 133). Let $\gamma:[0, \ell] \rightarrow \mathbb{R}^{2}$ be a parametrization by arc length of a simple curve $\mathcal{C}$. Assume that $\gamma$ is of class $\mathrm{C}^{2}$ and let $k(s)$ be its curvature at the point $\gamma(s)$. Then,

$$
\kappa(\gamma)=\int_{0}^{\ell}|k(s)| \mathrm{d} s
$$

For regular curves, therefore, the turn corresponds to the integral of the curvature (with respect to an arc-length parametrization).

The following property gives a lower bound of the turn for closed curves.

Property 3 (Fenchel's Theorem: [1], Theorem 5.1.5). For any Jordan curve $\mathcal{C}, \kappa(\mathcal{C}) \geq 2 \pi$. Moreover $\kappa(\mathcal{C})=2 \pi$ if and only if the interior of $\mathcal{C}$ is convex.

The interior of a Jordan curve is defined by the Jordan's curve Thoeorem: the interior of a Jordan curve $\mathcal{C}$ is the bounded connected component of $\mathbb{R}^{2} \backslash \mathcal{C}$.

The next property, known as Schur's Comparison Theorem, states that the distance between the ends of an arc is greater than the distance between the ends of another arc having same length but a greater turn. This property is useful for our purpose of defining local turns.

Property 4 (Schur's Comparison Theorem: [2], p. 150). Let $\gamma$ and $\bar{\gamma}$ be two simple curves parametrized by arc length on $[0, L]$ such that:

- $[\bar{\gamma}(0), \bar{\gamma}(L)] \cup \bar{\gamma}([0, L])$ is a convex Jordan curve, 


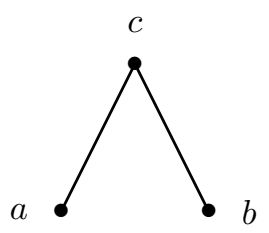

Figure 2: The turn of the $\operatorname{arcs} \mathcal{C}_{a}^{c}$ and $\mathcal{C}_{c}^{b}$ is zero but the turn of the $\operatorname{arc} \mathcal{C}_{a}^{b}$ is nonzero. Hence, triangle inequality fails with turns.

- for each subinterval $I \subset[0, L]$,

$$
\kappa(\gamma(I)) \leq \kappa(\bar{\gamma}(I)) .
$$

Then,

$$
\|\bar{\gamma}(L)-\bar{\gamma}(0)\| \leq\|\gamma(L)-\gamma(0)\| .
$$

Turn calculations sometimes require a kind of triangle inequality but in presence of angular points a strict statement of triangle inequality fails with turns as shown in Figure 2. Nevertheless, a loose version of the inequality can be derived from the following properties thanks to the existence of left and right tangents everywhere on a curve with finite turn.

We use a geometric definition of the left and right unit tangent vectors.

Definition 2 ([1], section 3.1). Let $(\mathcal{C}, \leq)$ be a geometric oriented curve.

- The unit vector $e_{l}(x)$ is the left unit tangent vector at $x$ if:

$$
\begin{array}{r}
\forall \epsilon>0, \exists y_{0} \in \mathcal{C}, y_{0}<x, \forall y \in \mathcal{C}, y_{0}<y \leq x, \\
\angle\left(x-y, e_{l}(x)\right)<\epsilon .
\end{array}
$$

- The unit vector $e_{r}(x)$ is the right unit tangent vector at $x$ if:

$$
\begin{array}{r}
\forall \epsilon>0, \exists y_{0} \in \mathcal{C}, y_{0}>x, \forall y \in \mathcal{C}, x \leq y<y_{0}, \\
\angle\left(y-x, e_{r}(x)\right)<\epsilon .
\end{array}
$$

- A curve having a right and a left unit tangent vector at each of its point is called one-sidedly smooth.

Property 5 ([1], Theorem 2.1.4, Theorem 3.1.1, Theorem 3.3.3 and Theorem 3.4.2). Let $\mathcal{C}$ be a one-sidedly smooth curve. Then, the set of angular points of $\mathcal{C}$ is countable, $\mathcal{C}$ is rectifiable and any arc-length parametrization $\gamma$ has both left-hand and right-hand derivatives $\gamma_{l}^{\prime}$ and $\gamma_{r}^{\prime}$. Moreover, for any $s \in[0, \mathcal{L}(C)]$, $\left\|\gamma_{l}^{\prime}(s)\right\|=\left\|\gamma_{r}^{\prime}(s)\right\|=1$.

Property 6 (Theorem 5.1.2 [1]). Every curve of finite turn is one-sidedly smooth. 
The existence of left and right tangent vectors makes it possible to split a curve into several parts using turns and tangent vectors.

Property 7 (Theorem 3.3.3 p. 53 and Theorem 5.1 .3 p. 122 [1]). Let $\mathcal{C}_{a}^{b}$ be an arc of $\mathcal{C}$ and $c$ be a point on $\mathcal{C}_{a}^{b}$. Let denote by $e_{l}(c)$ and $e_{r}(c)$ the left and right unit tangent vectors at $c$. The turn of $\mathcal{C}_{a}^{b}$ is finite if and only if the turns of $\mathcal{C}_{a}^{c}$ and $\mathcal{C}_{c}^{b}$ are both finite. In this case,

$$
\kappa\left(\mathcal{C}_{a}^{b}\right)=\kappa\left(\mathcal{C}_{a}^{c}\right)+\angle\left(e_{l}(c), e_{r}(c)\right)+\kappa\left(\mathcal{C}_{c}^{b}\right) .
$$

In the case where $a=b$ (C is closed), the previous equality becomes as follows.

$$
\kappa(\mathcal{C})=\angle\left(e_{l}(c), e_{r}(c)\right)+\kappa(\mathcal{C} \backslash\{c\}) .
$$

We immediately derive Corollary 1 (which is also valid if $\kappa(\mathcal{C} \backslash\{c\})=\infty$ ).

Corollary 1. Let $\mathcal{C}$ be a Jordan curve and c be a point in $\mathcal{C}$. Then, $\kappa(\mathcal{C} \backslash\{c\})>$ $\pi$.

Proof. The large inequality derives from Fenchel's Theorem 3 and the definition of geometric angles $(\angle(\mathbf{u}, \mathbf{v}) \in[0, \pi])$. The strict inequality is due to the fact that we cannot have both $\kappa(\mathcal{C})=2 \pi$ and $\angle\left(e_{l}(c), e_{r}(c)\right)=\pi$. Indeed, from Fenchel's Theorem, the former equality implies that $\mathcal{C}$ is the boundary of a convex body. Then, $\mathcal{C}$ has no cusp, that is $\angle\left(e_{l}(c), e_{r}(c)\right)<\pi$. As we did not find in the literature a proof of this last assertion, we propose one in Appendix A.

From Property 7, adding $\kappa\left(\mathcal{C}_{c}^{d}\right)$ in the right hand side of Equation $1\left(d \in \mathcal{C}_{c}^{b}\right)$, we easily derive the following corollary that will be used in the sequel.

Corollary 2. Let $\mathcal{C}$ be an oriented simple curve from a to b. Let $\mathcal{C}_{a}^{d}$ and $\mathcal{C}_{c}^{b}$ be two arcs of $\mathcal{C}$ that overlap with $a<c<d<b$. Then,

$$
\kappa\left(\mathcal{C}_{a}^{b}\right) \leq \kappa\left(\mathcal{C}_{a}^{d}\right)+\kappa\left(\mathcal{C}_{c}^{b}\right) .
$$

We end this section with a property linking the turn of a limit and the limit of the turns.

Property 8 ([1], Theorem 5.1.1 p. 120). If the curves $\left(\mathcal{C}_{m}\right)$ converge to the curve $\mathcal{C}$, then $\kappa(\mathcal{C}) \leq \liminf \kappa\left(\mathcal{C}_{m}\right)$.

In Property 8, " $\left(\mathcal{C}_{m}\right)$ converge to $\mathcal{C}$ " means that there exist parametrizations of the curves $\mathcal{C}_{m}$ that uniformly converge to a parametrization of $\mathcal{C}$ (see Section 1.4 in [1]).

\section{Locally turn-bounded curves}

Thanks to the notion of turn presented in Section 2, we define hereafter a new class of curves whose turn is locally bounded. Bounding the turn has the advantage of spatially constraining the curve with respect to any sufficiently tight 
sampling without imposing smoothness. Firstly, we will give some definitions (Definitions 3, 4, 5) and a few examples (Proposition 1) in order to help the reader to figure out the consequences of local constraints on turn. The impatient reader can skip this introduction to go to Proposition 2 which gives the operational characterization of the notion of locally turn bounded curve. Afterwards, Section 3 continues by establishing three easy propositions (Propositions 3 to 5) and a corollary (Corollary 4) that provide basic properties of the locally turn bounded curves. We end Section 3 by a lemma and a definition (Lemma 2 and Definition 6) that make it possible to distinguish the $\operatorname{arcs} \mathcal{C}_{a}^{b}$ and $\mathcal{C}_{b}^{a}$ under some assumptions.

Definition 3 (Turn-neighborhood). A point $b$ in $\mathcal{C}$ is in the turn-neighborhood of a point $a$ on a simple geometric curve (or on a Jordan curve) with angle $\theta$, if one of the $\operatorname{arcs}$ of $\mathcal{C}$ from $a$ to $b$ has a turn that is less than, or equal to $\theta$. The turn-neighborhood of the point $a$ on the geometric curve $\mathcal{C}$ with angle $\theta$ is denoted by $V_{\mathcal{C}}(a, \theta)$.

Figure 3 shows how the turn-neighborhood $V_{\mathcal{C}}(a, \theta)$ varies when changing the angle $\theta$ and the position of the point $a$.

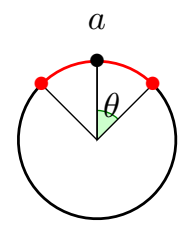

$\theta$

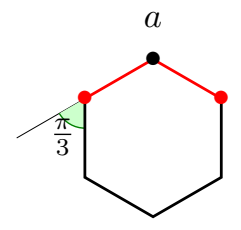

$\theta<\pi / 3$

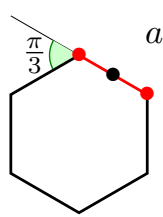

$\theta<\pi / 3$
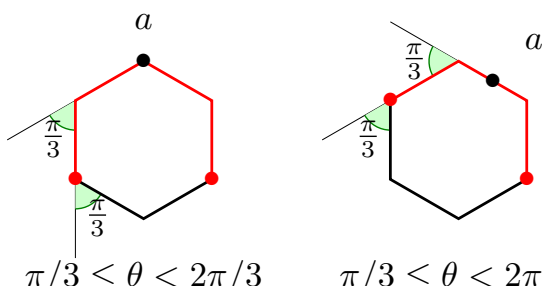

$\pi / 3 \leq \theta<2 \pi / 3 \quad \pi / 3 \leq \theta<2 \pi / 3$

Figure 3: The red arc is the turn-neighborhood $V_{\mathcal{C}}(a, \theta)$ of $a$ at different positions on the regular hexagon for the indicated $\theta$. On the circle, the turn-neighborhood remains the same, up to rotation, when changing the position of the point $a$.

Definition 4 (Turn step function). The turn step function $\theta \mapsto \sigma(\theta)$ is defined by

$$
\sigma(\theta):=\inf _{a \in \mathcal{C}} \mathrm{d}\left(a, \mathcal{C} \backslash V_{\mathcal{C}}(a, \theta)\right) .
$$

where d denotes the Euclidean distance. 
The turn step function $\theta \mapsto \sigma(\theta)$ is increasing. Indeed, for any $a \in \mathcal{C}$, the set $V_{\mathcal{C}}(a, \theta)$ increases (for the inclusion order) in function of $\theta$. Then, the distance from $a$ to the complement of $V_{\mathcal{C}}(a, \theta)$ increases too. If the turn of $\mathcal{C}$ is finite, there exists a value above which the turn step function has an infinite value for $V_{\mathcal{C}}(a, \theta)$ equals $\mathcal{C}$.

Proposition 1 (Examples). $\quad$ 1. The turn step function of a Jordan curve at 0 is 0 .

2. The turn step function of a convex Jordan curve at $\pi$ is $+\infty$.

3. The turn step function of a circle with radius $r$ at $\theta$ is $2 r \sin (\theta / 2)$ if $\theta<\pi$ and is infinite for $\theta \geq \pi$. (see Figure 4 and Figure 5).

4. The turn step function on a polygonal curve is a step function.

5. The turn step function on a regular $n$-gon $P_{n}$ inscribed in a circle of radius $r$ is

$$
\sigma_{P_{n}}(\theta)=\left\{\begin{array}{ll}
2 r \sin \left(\left\lfloor\frac{n \theta}{2 \pi}\right\rfloor \frac{\pi}{n}\right) & \text { if } \theta<\pi \\
+\infty & \text { otherwise }
\end{array} .\right.
$$

(see Figure 4 and Figure 5).

Proof. 1. Firstly, observe that, if $\mathcal{C}$ is a polygon, taking points $a$ arbitrarily closed to a vertex, we have $\mathrm{d}\left(a, \mathcal{C} \backslash V_{\mathcal{C}}(a, 0)\right)$ arbitrarily small, that is $\sigma(0)=0$. By contradiction, now assume that $\sigma(0)=c>0$ for some Jordan curve $\mathcal{C}$. Then, thanks to the compacity of $\mathcal{C}$, we can cover the whole curve $\mathcal{C}$ with a finite set of balls $B\left(a_{i}, c\right), 1 \leq i \leq n$. By definition of $c$, the turns $\kappa\left(\mathcal{C} \cap B\left(a_{i}, c\right)\right)$ are reduced to the angles between the left and right tangent vectors at $a_{i}: \mathcal{C}$ is a polygon. Contradiction.

2. According to Fenchel's Theorem 3 and Property 7, for any point on a convex Jordan curve, the $\pi$-neighborhood is the whole curve.

3. The $\theta$-neighborhood of any point $a$ of a circle of radius $r$ is an arc of circle of length $\theta r$ centered in $a$. The distance between the point $a$ and the rest of the circle is $2 r \sin (\theta / 2)$ (see Figure 4).

4. The turn between two points on a polygonal curve is a finite sum of geometric angles.

5. The $\theta$-neighborhood are described in Figure 3. The $\theta$-neighborhood of a vertex $a$ of $P_{n}$ is made of the $2\left(\left\lfloor\frac{n \theta}{2 \pi}\right\rfloor+1\right)$ nearest sides of $P_{n}$. The $\theta-$ neighborhood of a point $a$ on an open edge of $P_{n}$ is made of the $2\left\lfloor\frac{n \theta}{2 \pi}\right\rfloor+1$ nearest sides of $P_{n}$. Then the distance between $a$ and a point outside $V_{P_{n}}(a, \theta)$ is minimal when $a$ lies in an open side. Observe that, in this latter case, the $\theta$-neighborhood does not depend on the position of $a$ in the open side. Then, $a$ can be arbitrarily close to a vertex (see Figure 4 ). Hence, for $\theta<\pi$,

$$
\sigma(\theta)=2 r \sin \left(\left\lfloor\frac{\theta}{2 \pi / n}\right\rfloor \frac{\pi}{n}\right) .
$$


Figure 4 illustrates the definition of the turn step with different curves. In Figure 5, we plot the turn step functions of circles and regular polygons.

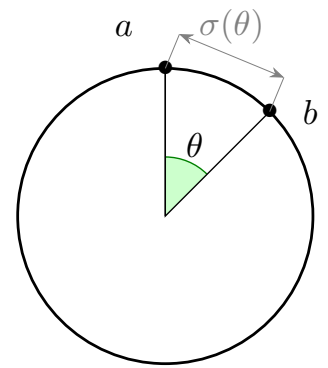

$$
\sigma(\theta)=2 r \sin \left(\frac{\theta}{2}\right)
$$

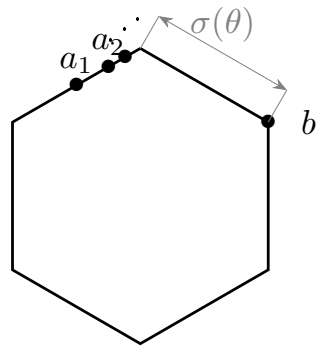

$\theta=\pi / 3$

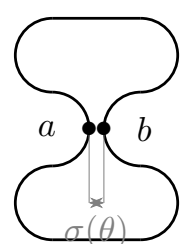

$\theta=\pi / 2$

Figure 4: For the chosen value of $\theta$, the corresponding $\theta$-turn-step $\sigma(\theta)$. On the hexagon, the distance between a point and one end of its $\theta$-neighborhood depends on the position of the point. This distance is asymptotically achieved by a sequence of points $\left(a_{i}\right)$ lying on one side which tends to a corner of the hexagon. The end of the $\theta$-neighborhood of each $a_{i}$ is the point $b$. Notice that the $\theta$-turn step is not always the distance between a point and the end of the $\theta$-neighborhood. The third curve is a counterexample to this wrong assumption.

Definition 5 (Locally turn-bounded curves). Let $\theta \geq 0, \delta \geq 0$. A Jordan curve $\mathcal{C}$ is $(\theta, \delta)$-locally turn-bounded if, for any $a \in \mathcal{C}$, the Euclidean distance from $a$ to $\mathcal{C} \backslash V_{\mathcal{C}}(a, \theta)$ is greater than, or equal to $\delta$ :

$$
\sigma(\theta) \geq \delta .
$$

In the rest of the article, we will shorten $(\theta, \delta)$-locally turn-bounded curve by $(\theta, \delta)$-LTB curve.

Remark 3. Local turn-boundedness is scale invariant: let $\mathcal{C}$ be a $(\theta, \delta)$-LTB Jordan curve. Then, the curve $k \mathcal{C}, k>0$, is $(\theta, k \delta)$-LTB. It is a direct consequence of Remark 2.

The following proposition explains how to apply the notion of local turnboundedness to a concrete geometric configuration.

Proposition 2 (Characteristic property of local turn-boundedness). The curve $\mathcal{C}$ is $(\theta, \delta)-L T B$ if and only if, for any two points a and $b$ in $\mathcal{C}$ such that $\mathrm{d}(a, b)<$ $\delta$, the turn of one of the arcs of the curve $\mathcal{C}$ delimited by $a$ and $b$ is less than or equal to $\theta$. 

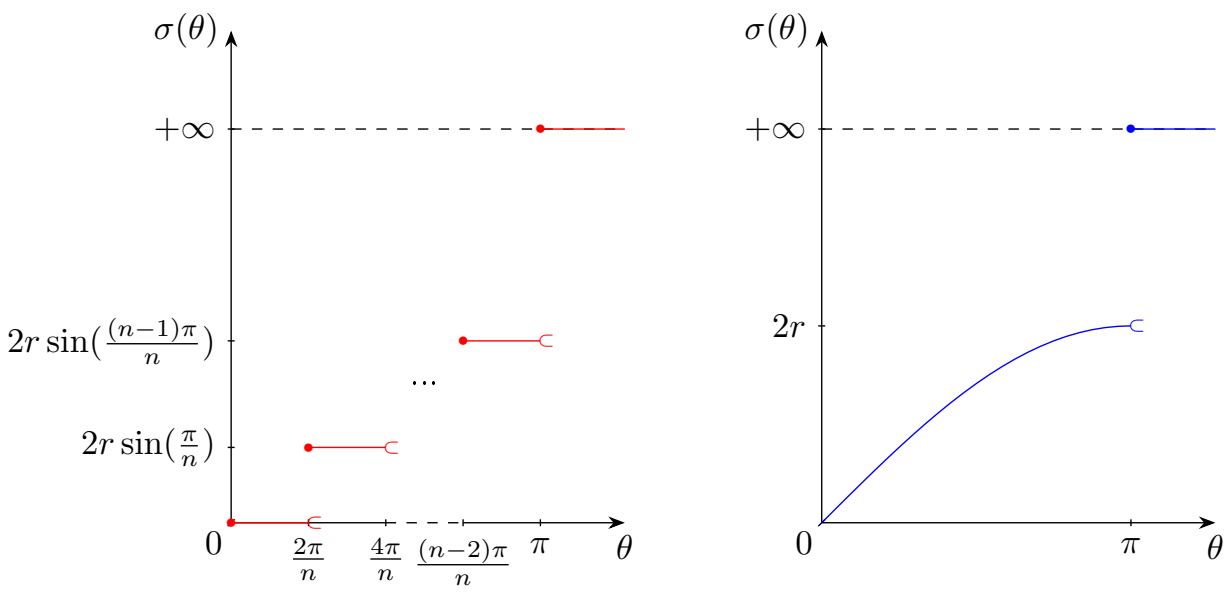

Figure 5: On the left (red), the graph of the turn-step function $\sigma$ for a regular $n$-gon inscribed in a circle of radius $r$, on the right (blue), the graph of the turn-step function $\sigma$ for a circle.

Proof. This property is just a contrapositive statement of Definition 5. Indeed,

$$
\begin{aligned}
\mathcal{C} \text { is }(\theta, \delta) \text {-LTB } & \Longleftrightarrow \forall a \in \mathcal{C}, \delta \leq d\left(a, \mathcal{C} \backslash V_{\mathcal{C}}(a, \theta)\right) \\
& \Longleftrightarrow \forall a \in \mathcal{C}, \forall b \notin V_{\mathcal{C}}(a, \theta), \delta \leq d(a, b) \\
& \Longleftrightarrow \forall a \in \mathcal{C}, \forall b \in \mathcal{C}, \\
& \kappa\left(\mathcal{C}_{a}^{b}\right)>\theta \text { and } \kappa\left(\mathcal{C}_{b}^{a}\right)>\theta \Longrightarrow \delta \leq d(a, b) \\
& \Longleftrightarrow \forall a \in \mathcal{C}, \forall b \in \mathcal{C}, \\
& d(a, b)<\delta \Longrightarrow \kappa\left(\mathcal{C}_{a}^{b}\right) \leq \theta \text { or } \kappa\left(\mathcal{C}_{b}^{a}\right) \leq \theta .
\end{aligned}
$$

Using the previous characteristic property, let us now show that the class of LTB curves contains the smooth curves of class $\mathrm{C}^{2}$.

Corollary 3. Jordan curves of class $\mathrm{C}^{2}$ are $\left(\theta, 2 r_{\mathcal{C}} \sin (\theta / 2)\right)-L T B$ for any $\theta \leq \pi$, $r_{\mathcal{C}}$ being the minimum radius of curvature of $\mathcal{C}$.

Proof. Let $\mathcal{C}$ be a Jordan curve of class $\mathrm{C}^{2}$. Then, $\mathcal{C}$ has an arc length parametrization $\gamma$ and the absolute value of its curvature is bounded from above by the real $1 / r_{\mathcal{C}}$. By Property 2 , the turn of $\mathcal{C}$ between two points is bounded by $\frac{s}{r_{\mathcal{C}}}$ where $s$ is the geodesic distance between the two points. Considering a circle $D$ of radius $r_{\mathcal{C}}$, we derive that the turn of $\mathcal{C}$ between two points $a, b$ at geodesic distance $\ell$ less than $r_{\mathcal{C}} \pi$ is less than the turn of $D$ between two points $c, d$ at geodesic distance $\ell$. Hence, Schur's Comparison Theorem applies: $\|a-b\| \geq\|c-d\|$ and 


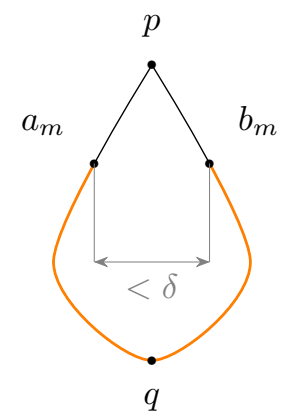

Figure 6: The sequences of points of $\mathcal{C},\left(a_{m}\right)$ and $\left(b_{m}\right)$ tends to the point $p$ on both sides of $p$. The curve $\left(C_{m}\right)$ is drawn in orange.

since

$$
\|c-d\|=2 r_{\mathcal{C}} \sin \left(\frac{l}{2 r_{\mathcal{C}}}\right) \geq 2 r_{\mathcal{C}} \sin \left(\frac{\kappa\left(\mathcal{C}_{a}^{b}\right)}{2}\right)
$$

(for $\left.\ell /\left(2 r_{\mathcal{C}}\right) \leq \pi / 2\right)$, it follows that, for any $\theta \in(0, \pi], \kappa\left(\mathcal{C}_{a}^{b}\right) \leq \theta$ whenever $\|a-b\|<2 r_{\mathcal{C}} \sin (\theta / 2)$. Then, according to Proposition 2, the result holds.

Intuitively, local turn-boundedness must also be a punctual turn-boundedness. This is verified just hereafter.

Proposition 3. Let $\mathcal{C}$ be a $(\theta, \delta)$-LTB curve where $\theta<\pi$. Then, for any point $p \in \mathcal{C}$, one has $\angle\left(e_{l}(p), e_{r}(p)\right) \leq \theta$ where $e_{l}(p)$ and $e_{r}(p)$ denote respectively the left and right tangent vectors at point $p$.

Proof. The notations of the proof are summed up in Figure 6. Let $\mathcal{C}$ be a $(\theta, \delta)$ LTB curve where $\theta<\pi$ and $p$ be a point in $\mathcal{C}$. Let $q \neq p \in \mathcal{C}$ and $\left(a_{m}\right)$, resp. $\left(b_{m}\right)$ be a sequence of points in $\mathcal{C}$ such that $\left(a_{m}\right) \rightarrow p,\left(b_{m}\right) \rightarrow p$ and $a_{m} \in \mathcal{C}_{p}^{q}$ while $b_{m} \in \mathcal{C}_{q}^{p}$ for any $m$. Then, from Corollary $1, \kappa(\mathcal{C} \backslash\{p\})>\pi$. Moreover, from Property $8, \kappa(\mathcal{C} \backslash\{p\}) \leq \liminf \kappa\left(\mathcal{C}_{m}\right)$ where $\mathcal{C}_{m}$ is the arc between $a_{m}$ and $b_{m}$ included in $\mathcal{C} \backslash\{p\}$. Thus, on the one hand, there exists $m_{0}$ such that, for any $m>m_{0}, \kappa\left(\mathcal{C}_{m}\right)>\pi$. On the other hand, there clearly exists $m_{1}>m_{0}$ such that $\mathrm{d}\left(a_{m}, b_{m}\right)<\delta$ for any $m>m_{1}$. Let $m>m_{1}$. As $\mathcal{C}$ is $(\theta, \delta)$-LTB and $\kappa\left(\mathcal{C}_{m}\right)>\pi$, we derive that $\kappa\left(\mathcal{C} \backslash \mathcal{C}_{m}\right) \leq \theta$. We conclude, thanks to Property 7 , that $\angle\left(e_{l}(p), e_{r}(p)\right) \leq \theta$.

From Proposition 3 , we derive that a $(\theta, \delta)$-LTB polygon has inner angles greater than or equal to $\pi-\theta$. Provided that $\delta$ is not greater than any edge of the polygon and any distance between non-consecutive edges, this last property is a sufficient condition as well. Indeed, with such a value for $\delta$, points at distance less than $\delta$ belong to the same edge or to two consecutive edges. Thereby, they are linked by an arc whose turn is at most $\theta$. Proposition 3 also shows that LTB-curves fill a gap between smooth curves and unconstrained polygons: they may have angular points but not too much sharp. 


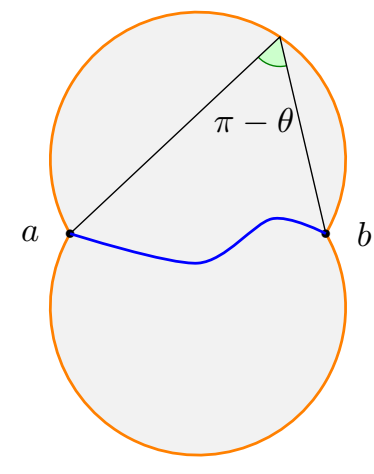

$\theta=2 \pi / 3$

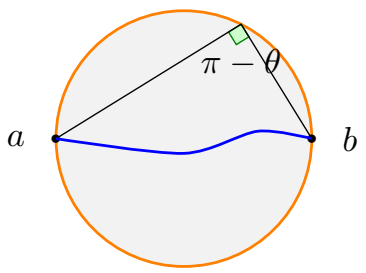

$\theta=\pi / 2$

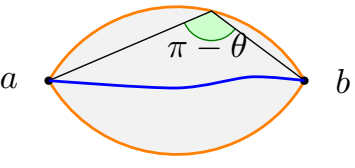

$\theta=\pi / 3$

Figure 7: Illustration of Proposition 4 for three values of the parameter $\theta: \pi / 3$, $\pi / 2,2 \pi / 3$. Given two points $a, b \in \mathcal{C}$ such that $\mathrm{d}(a, b)<\sigma(\theta)$, then one of the arc of $\mathcal{C}$ between $a$ and $b$ belongs to the grey area.

The next proposition makes it possible to localize a locally turn-bounded curve from a sufficiently tight sampling. Figure 7 illustrates the proposition.

Proposition 4. Let $\mathcal{C}$ be a simple $(\theta, \delta)$-LTB curve. Let $a, b$ be two points on $\mathcal{C}$ such that $\mathrm{d}(a, b)<\delta$. Then, the arc of $\mathcal{C}$ delimited by $a$ and $b$ of smallest turn is included in the union of the two truncated closed disks where the line segment $[a, b]$ is seen from an angle greater than or equal to $\pi-\theta$.

Proof. Since $\mathrm{d}(a, b)<\delta$, by Proposition 2, the turn of one of the $\operatorname{arcs}$ of $\mathcal{C}$ between $a$ and $b$ is less than or equal to $\theta$. Denote by $\mathcal{C}_{0}$ such an arc. Let $c$ be a point on $\mathcal{C}_{0}$. By definition, the turn of the polygonal line $[a, c, b]$ is less than or equal to the turn of $\mathcal{C}_{0}$. Then the geometric angle $\widehat{a c b}$ is greater than or equal to $\pi-\theta$. We conclude the proof by invoking the inscribed angle theorem.

In Figure 8, we use Proposition 4 to localize a $(\pi / 2, \delta)$-LTB curve from a sufficiently tight sampling of the curve with respect to $\delta$.

Proposition 5 states that LTB curves for angles $\theta \leq \pi / 2$ are locally pathconnected subsets of the Euclidean plane. Locally path-connectedness can be seen as a thickness property. Indeed, locally path-connectedness implies that dilating a LTB curve by a sufficiently small ball (here, with radius less than $\delta / 2$ ) does not change the homotopy type of the curve (no connected component of the interior or the exterior of the curve is created).

Proposition 5. Let $\mathcal{C}$ be a $(\theta, \delta)-L T B$ Jordan curve with $\theta \in(0, \pi / 2]$ and $a \in \mathcal{C}$. Then, for any $\epsilon \leq \delta$, the intersection of $\mathcal{C}$ with the open disk $B(a, \epsilon)$ is pathconnected and is therefore an arc of $\mathcal{C}$. Furthermore, the turn of this arc is less than or equal to $3 \theta$. 


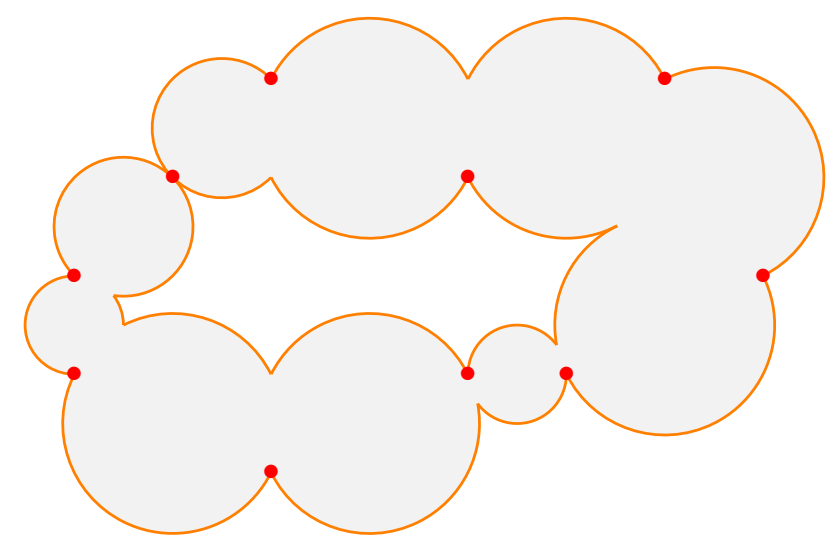

Figure 8: Any $(\pi / 2, \delta)$-LTB curve sampled by the set of red points is localized in the gray region delimited by the two orange curves provided that the distance between two consecutive sampling points is less than $\delta$.

Proof. The notations of the proof are summed up in Figure 9. Let $a \in \mathcal{C}$. Let $b_{1}, b_{2} \in \mathcal{C} \cap B(a, \epsilon)$. Then, by Proposition 2, the turn of one of the $\operatorname{arcs}$ of $\mathcal{C}$ between $a$ and $b_{1}$, resp. between $a$ and $b_{2}$, is less than or equal to $\theta$. This arc is denoted by $\mathcal{C}_{a}^{b_{1}}$, resp. $\mathcal{C}_{a}^{b_{2}}$. So, from Proposition 4 and for $\theta \leq \pi / 2$, this arc is included in the disk with diameter $\left[a, b_{1}\right]$, resp. $\left[a, b_{2}\right]$, which is itself included in $B(a, \epsilon)$. Hence, $\mathcal{C} \cap B(a, \epsilon)$ is path-connected. Furthermore, we derive from Property 7 that

$$
\begin{aligned}
\kappa\left(\mathcal{C}_{b_{1}}^{b_{2}}\right) & \leq \kappa\left(\mathcal{C}_{b_{1}}^{a}\right)+\kappa\left(\mathcal{C}_{a}^{b_{2}}\right)+\angle\left(e_{l}(a), e_{r}(a)\right) \\
& \leq 2 \theta+\angle\left(e_{l}(a), e_{r}(a)\right) .
\end{aligned}
$$

By Proposition $3, \angle\left(e_{l}(a), e_{r}(a)\right) \leq \theta$, then $\kappa\left(\mathcal{C}_{b_{1}}^{b_{2}}\right) \leq 3 \theta$.

Observe that for $\pi / 2<\theta<\pi$, in particular for polygons with acute angles, Proposition 5 does not hold (the intersection of the curve with a ball near an acute angle may have two connected components).

The rectifiability of a $(\theta, \delta)$-LTB curve is a consequence of Proposition 5 .

Corollary 4. $A(\theta, \delta)-L T B$ curve with $\theta \in(0, \pi / 2]$ has a finite turn and is thus rectifiable.

Proof. Let $\mathcal{C}$ be a $(\theta, \delta)$-locally turn-bounded curve. The open balls $B(a, \delta / 2)$, $a \in \mathcal{C}$, cover the compact set $\mathcal{C}$. Then, there exists a finite subset of $\mathcal{C}$, $\left\{a_{0}, \ldots, a_{m}\right\}$ such that $\bigcup_{i=0}^{m} B\left(a_{i}, \delta / 2\right)$ covers $\mathcal{C}$. By Proposition 5 , for each $i, \mathcal{C} \cap B\left(a_{i}, \delta / 2\right)$ is an arc of $\mathcal{C}$ whose turn is less than, or equal to $3 \theta$.

Since the balls are open and thus overlap, by Corollary $2, \kappa(\mathcal{C}) \leq \sum_{i=0}^{m} \kappa(\mathcal{C} \cap$ $\left.B\left(a_{i}, \delta / 2\right)\right)$. Therefore, $\kappa(\mathcal{C}) \leq(m+1)(3 \theta)$. 


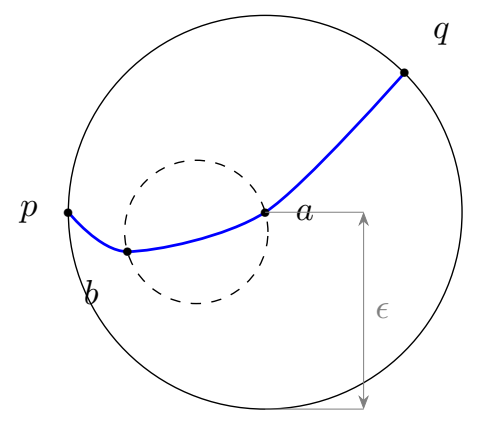

Figure 9: In blue, the $\operatorname{arc} \mathcal{C}_{p}^{q}$ which is the intersection between $\mathcal{C}$ and the disk $B(a, \epsilon)$. The $\operatorname{arc} \mathcal{C}_{a}^{b}$ is included in the disk of diameter $[a, b]$ delimited by the dashed circle.

From Corollary 4, we derive that LTB curves are one-sidedly smooth (Property 6) and contain at most countably many angular points (Property 5). We also deduce from Corollary 4 that the class of LTB curves contains no fractal curve. This is not satisfactory in a multi-resolution context. Nevertheless, local turn boundedness is a step between smooth and fully realistic models in multi-resolution environments.

Because of the strict inequality $(\mathrm{d}(a, b)<\delta)$ in the characteristic property of local turn-boundedness (Proposition 2), it could be necessary to deal with parameters $\delta$ greater than the diameter ${ }^{1}$ of the curve. The next lemma shows that it is actually not necessary (the proof, somewhat technical, is given in Appendix B).

Lemma 1. Let $\mathcal{C}$ be $a(\theta, \delta)-L T B$ curve with $\theta<2 \pi / 3$. Then,

$$
\delta \leq \operatorname{diam}(\mathcal{C})
$$

where diam denotes the diameter.

Using the characteristic property stated in Proposition 2, one of the main difficulties is that there is no way to know which of the two arcs between two points at distance less than $\delta$ has its turn less than $\theta$.

When $\theta \leq \frac{\pi}{2}$, the next lemma removes any ambiguity.

Lemma 2. Let $\theta \in(0, \pi / 2]$ and $\mathcal{C}$ be $a(\theta, \delta)-L T B$ curve. For any $a, b \in \mathcal{C}$ such that $0<\mathrm{d}(a, b)<\delta$, there exists a unique arc of $\mathcal{C}$ from a to $b$ whose turn is less than or equal to $\frac{\pi}{2}$.

\footnotetext{
${ }^{1}$ Diameter of a set $S:$ supremum of the set of all distances between pairs of points in $S$.
} 
Proof. We prove a contrapositive statement. Let $\mathcal{C}$ be a $(\theta, \delta)$-LTB curve where $\theta \in(0, \pi / 2]$ and let $a, b$ be two points in $\mathcal{C}$ such that $0<\mathrm{d}(a, b)<\delta$ and $\mathcal{C}_{a}^{b}$, $\mathcal{C}_{b}^{a}$ both have a turn less than or equal to $\frac{\pi}{2}$. By Proposition $4, \mathcal{C}_{a}^{b}$ and $\mathcal{C}_{b}^{a}$ are included in the disk of diameter $[a, b]$ which then contains the whole curve $\mathcal{C}$. Thus, the diameter of $\mathcal{C}$ is smaller than $\delta$. Contradiction with Lemma 1.

Thanks to Lemma 2, we can now define the straightest arc between two close points of a $(\theta, \delta)$-LTB curve when $\theta \leq \pi / 2$.

Definition 6 (Straightest arc between two points). Let $\theta \in(0, \pi / 2]$ and $\mathcal{C}$ be a $(\theta, \delta)$-LTB curve. Between two distinct points at distance less than $\delta$, the unique arc whose turn is less than or equal to $\theta$ is called the straightest arc between a and $b$.

\section{Properties of locally turn-bounded curves re- lated to the Gauss digitization}

The aim of this section is to establish the following theorem about Gauss digitization of LTB Jordan curves on fine enough grids.

Theorem 1. Let $\mathcal{C}$ be $(\theta, \delta)-L T B$ curve with $\theta \leq \pi / 2$ and $h$ be a grid step compatible with $\mathcal{C}$. Then, the Gauss digitization of $\mathcal{C}$ for the grid step $h$ is a Jordan curve whose interior is 4-connected.

Firstly, we will recall what is the Gauss digitization of a set and how we define the Gauss digitization of a Jordan curve. We will also recall the notion of well-composedness which expresses the manifoldness of a digitized shape, more precisely, of an union of pixels. Pixels we are dealing with are mainly squares. Nevertheless, when more general pixels (regular tiles, or even compact tiles) can be used for free in the proofs, we will give general statements in the propositions. As a first step towards the proof of Theorem 1, we will describe the intersection of a LTB-curve with a pixel. Actually, studying straightest arcs starting and ending in a given tile, we will show that such arcs are generally not entirely included in the tile but in a swollen tile (Definition 7 and Proposition 6). The next step will be to define and describe the supremum (for the inclusion) actually a maximum - of all the arcs starting and ending in a given tile $T$. We will call it arc passing through $T$ (Definition 8 and Proposition 7 ). The assumptions under which all the previous results are valid will be gathered in the notion of grid step compatible with a given LTB-curve (Definition 9). The last step of this effort towards a topological description of the manner the curve separates the grid points will be accomplished by considering dual pixels, that is unit squares whose vertices are grid points: the end points of the arc passing through a dual pixel $T$ determines the membership of the vertices of $T$ to the interior or the exterior of the LTB-curve (Lemma 4 and Proposition 8). Finally, we will state and prove Theorem 1 in two parts: well-composedness (Proposition 9) and 4-connectedness (Corollary 5). 

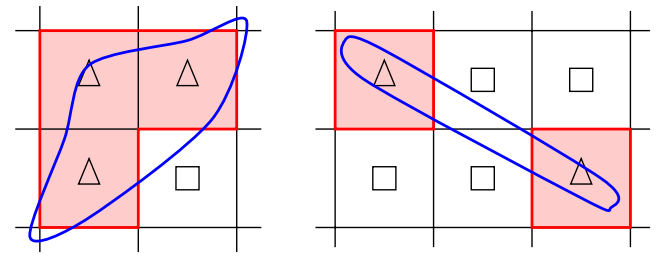

$-\mathcal{C} \quad-\partial_{h}(\mathcal{C}) \quad \triangle$ point inside $\mathcal{C}$

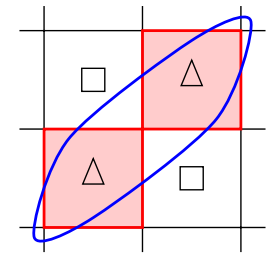

point outside $\mathcal{C}$

Figure 10: Gauss digitizations in red of continuous shape delimited by a Jordan curve $\mathcal{C}$ in blue . Left and center: well-composed. Right: non wellcomposed.

Let $h>0$ be a sampling grid step, the Gauss digitization of a shape $S$ is defined as $S \cap(h \mathbb{Z})^{2}$. By abuse of language, given a Jordan curve $\mathcal{C}$ which is the border of the compact shape $S$, we define its Gauss digitization - we write $\partial_{h}(\mathcal{C})$ - as the border of the union of the squares $p \oplus([-h / 2, h / 2] \times[-h / 2, h / 2])$ where $\oplus$ denotes the Minkowski sum and $p \in S \cap(h \mathbb{Z})^{2}$. The Gauss digitization of $\mathcal{C}$ is well-composed if it is a disjoint union of Jordan curves (see Figure 10).

The information on the turn makes it possible to define a domain where the arc of smallest turn of a $(\theta, \delta)$-LTB curve passing through a tile of the grid is lying (Definition 7 ). Before that, we need to prove a technical lemma that will be used in the proofs of Proposition 6 and Proposition 9.

Lemma 3. Let $\mathcal{C}$ be a curve with endpoints $a, b$ such that the straight segment $(a, b)$ does not intersect the curve $\mathcal{C}$. Let $P$ be a polygonal line from a to $b$ such that $P \backslash\{a, b\}$ lies in the interior of the Jordan curve $\mathcal{C} \cup[a, b]$ and $P \cup[a, b]$ is convex. Then $\kappa(\mathcal{C})>\kappa(P)$.

Proof. We set $P=\left[a, p_{1}, \ldots, p_{m}, b\right]$. Let $c$ be any point in $(a, b)$ and $Q=\left[a, q_{1}\right.$, $\left.\ldots, q_{m}, b\right]$ be the polygonal line obtained by projecting from the point $c$ on the curve $\mathcal{C}$ the polygon $P$ (see Figure 11). By projection of a point $x$, we mean the first intersection point $y$ between $\mathcal{C}$ and the half-line $D$ starting from $c$ and directed by $x-c$. This intersection exists and is well defined for $P \backslash\{a, b\}$ lies in the interior of $\mathcal{C} \cup[a, b]$ and $\mathcal{C} \cap D$ is a compact set. Note that we do not assert that the point $q_{i}$ is the projection of the point $p_{i}$ but we claim that the polyline $P$ deprived of its endpoints lies in the interior of the polygon $Q \cup[a, b]$ and the polyline $Q$ is inscribed in $\mathcal{C}$. Then, $\kappa(\mathcal{C}) \geq \kappa(Q)$ by definition of $\kappa(\mathcal{C}), \kappa(Q \cup[b, a]) \geq \kappa(P \cup[b, a])$ by Fenchel's Theorem (Property 3) and $\angle\left(a-b, p_{1}-a\right)>\angle\left(a-b, q_{1}-a\right), \angle\left(a-b, b-p_{m}\right)>\angle\left(a-b, b-q_{m}\right)$ for $P$ is inside $Q \cup[a, b]$. Since $\kappa(P \cup[b, a])=\kappa(P)+\angle\left(a-b, p_{1}-a\right)+\angle\left(a-b, b-p_{m}\right)$ and $\kappa(Q \cup[b, a])=\kappa(Q)+\angle\left(a-b, q_{1}-a\right)+\angle\left(a-b, b-q_{m}\right)$ by definition of the turn of a polygon, the result holds.

Let us now define the "swollen" tile in which lies an arc of a LTB-curve 


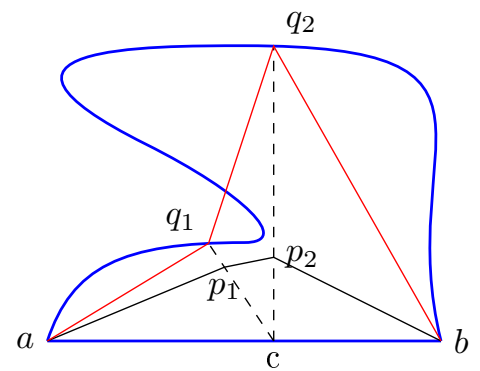

Figure 11: Blue: the curve $\mathcal{C}$ and the line segment $[a, b]$. Black: the polygonal line $P=\left[a, p_{1}, p_{2}, b\right]$. Black, dashed: the projection of $p_{1}$ and $p_{2}$ on $\mathcal{C}$ yields the points $q_{1}$ and $q_{2}$. Red: the polygonal line $Q=\left[a, q_{1}, q_{2}, b\right]$.

passing through a tile of the plane under some hypotheses.

Definition 7 (Swollen set). Let $P$ be a polygon $\left[p_{0}, \ldots, p_{N}\right]$ with $p_{0}=p_{N}$ and $A$ be the interior of $P$. The $\theta$-swollen set of $P$ denoted by $\hat{P}_{\theta}$ is defined by:

$$
\hat{P}_{\theta}:=P \cup A \cup \bigcup_{k=0}^{N-1} D_{k}
$$

with $D_{k}$ the truncated closed disk outside $P$ where the segment $\left[p_{k}, p_{k+1}\right]$ is seen from an angle greater than or equal to $\pi-\theta$. Moreover $\hat{P}_{\frac{\pi}{2}}$ is shorten by $\hat{P}$.

The notion of swollen set is illustrated in Figure 12 and in Figure 16.

Proposition 6. Assuming an n-regular tiling of the plane with edge length $h$ $(n \in\{3,4,6\})$, let $\mathcal{C}$ be a $(\theta, \delta)$-LTB Jordan curve with $\theta \leq 2 \pi / n$ and $\delta>$ $h \sqrt{n-2}$. Let $T$ be a tile crossed by $\mathcal{C}$ and $a, b$ be two points of $T \cap \mathcal{C}$. Then, the arc $\mathcal{C}_{a}^{b}$ of $\mathcal{C}$ of smallest turn delimited by $a$ and $b$ lies in the $\theta$-swollen set of $T$. In particular, the maximum distance between a point of $\mathcal{C}_{a}^{b}$ and $T$ is bounded from above by $\frac{h}{2} \tan \left(\frac{\theta}{2}\right)$.

Proof. Let $\mathcal{C}_{a}^{b}$ be the arc of $\mathcal{C}$ of smallest turn delimited by $a$ and $b$. As the diameter of $T$ is $h \sqrt{n-2}$, by the hypothesis $\delta>h \sqrt{n-2}$ and since $\mathcal{C}$ is a $(\theta, \delta)$-LTB curve, one has $\kappa\left(\mathcal{C}_{a}^{b}\right) \leq \theta \leq 2 \pi / n$. Let $o$ be a point lying on $\mathcal{C}_{a}^{b}$ and outside $T$, if any. Let $\mathcal{C}_{c}^{d}$ be the closure of the connected component of $\mathcal{C}_{a}^{b} \backslash T$ containing the point $o$. Notice that $c$ and $d$ are on the border $\partial T$ of $T$. Indeed if $c$ or $d$ is in the interior of $T$, then there exist points of $\mathcal{C}_{c}^{d}$ inside $T$ and if $c$ or $d$ are outside $T$, there exists a connected component of $\mathcal{C}_{a}^{b} \backslash T$ properly containing $\mathcal{C}_{c}^{d}$.

We claim that the point $d$ belongs to the same edge as $c$. Indeed, if it was not the case, one of the two polygonal lines from $c$ to $d$ in the boundary of $T$ would lie in the interior of the Jordan curve $\mathcal{C}_{c}^{d} \cup[c, d]$ and would contain at least a vertex of $T$. Then, according to Lemma 3 , the turn of the subarc $\mathcal{C}_{c}^{d}$ of $\mathcal{C}_{a}^{b}$, 
would be greater than the turn at a vertex of $T$, that is $2 \pi / n$. A contradiction. Hence, $c$ and $d$ belong to the same edge. By Proposition 4, we derive that $\mathcal{C}_{c}^{d}$ lies in the union of the two truncated disks where the segment $[c, d]$ is seen from an angle greater than or equal to $\pi-\theta$. One of these truncated disks is included in $T$ while the other, exterior to $T$, is included in the swollen set $\hat{T}_{\theta}$. Hence $o$ lies in the swollen set $\hat{T}_{\theta}$ whose Hausdorff distance to $T$ is $\frac{h}{2} \tan \left(\frac{\theta}{2}\right)$.

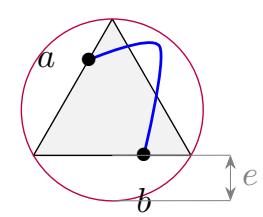

$\theta=\frac{\pi}{3}, \delta>h$

$e=h(\sqrt{3} / 6)$

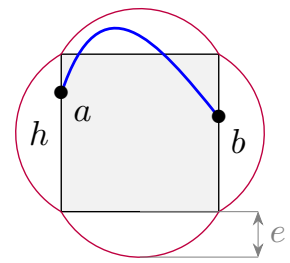

$\theta=\frac{\pi}{3}, \delta>\sqrt{2} h$ $e=h(\sqrt{3} / 6)$

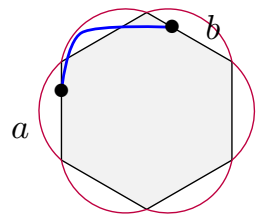

$$
\theta=\frac{\pi}{4}, \delta>2 h
$$$$
e=h(\sqrt{2}-1) / 2
$$

Figure 12: Gray: a tile $T$ with edge length $h$. Blue, thick: a LTB curve arc with ends in $T$. Red: the boundary of the swollen set $\hat{T}_{\theta}$. The Hausdorff distance between $\hat{T}_{\theta}$ and the tile $T$ is $e$.

When $\theta<\pi / 2$, Proposition 6 makes it possible to localize the straightest arc between any two points of a a sufficiently small tile. Nevertheless, we still need to define the minimal straightest arc including the whole intersection between $\mathcal{C}$ and $T$.

Definition 8 (Arc passing through T). Let $\theta \in(0, \pi / 2]$ and $\mathcal{C}$ be a $(\theta, \delta)$-LTB Jordan curve . Let $T$ be a closed set whose diameter is strictly less than $\delta$. The arc of $\mathcal{C}$ passing through $T$ denoted by $\mathcal{C}_{T}$ is defined by

$$
\mathcal{C}_{T}:=\bigcup_{a, b \in T \cap \mathcal{C}} \mathcal{C}_{a}^{b}
$$

where $\mathcal{C}_{a}^{b}$ is the straightest arc between $a$ and $b$.

We now show some properties of the arc passing through a tile provided this tile is sufficiently small compared to $\mathcal{C}$ : it is a straightest arc between some points in $T$ (hence its turn is less than or equal to $\frac{\pi}{2}$ ) and it is maximal for this property. Furthermore, its complementary in $\mathcal{C}$ does not intersect $T$.

Proposition 7. Let $\theta \in(0, \pi / 2]$ and $\mathcal{C}$ be $a(\theta, \delta)$-LTB Jordan curve. Let $d$ be the diameter of $\mathcal{C}$. Let $T$ be a closed set included in an open disk $B(c, r)$ with $r$ less than or equal to $\min \left(\frac{1}{2} \delta, \frac{\sqrt{2}}{4} d\right)$. Then, the arc $\mathcal{C}_{T}$ passing through $T$ is the unique arc of $\mathcal{C}$ of turn less than or equal to $\frac{\pi}{2}$ having its end points in $T$ and such that the straightest arc between any two points of $T$ is included in $\mathcal{C}_{T}$. Moreover,

$$
\left(\mathcal{C} \backslash \mathcal{C}_{T}\right) \cap T=\emptyset .
$$


Proof. Since the proof is somewhat long and tedious, we put it in Appendix D.

Going back to the main case where pixels are square tiles, we propose the following definition that corresponds to the hypotheses of Proposition 7 .

Definition 9 (Compatibility hypothesis). A grid with step $h$ or a square of side length $h$ is said to be compatible with the curve $\mathcal{C}$ if the following conditions are fulfilled:

1. the curve $\mathcal{C}$ is $(\theta, \delta)$-locally turn-bounded with $\theta \in\left(0, \frac{\pi}{2}\right]$,

2. $h$ is strictly smaller than $\min \left(\frac{\sqrt{2}}{2} \delta, \frac{1}{2} \operatorname{diam}(\mathcal{C})\right)$.

Lemma 4 and Proposition 8 investigate the positions of the vertices of a square pixel relatively to the arc passing through this pixel.

Lemma 4. Let $\mathcal{C}$ be a $(\theta, \delta)-L T B$ Jordan curve with $\theta \leq \frac{\pi}{2}$ and $T$ be a square compatible with $\mathcal{C}$. If $\mathcal{C}$ contains a vertex $v$ of $T$ then either this vertex $v$ is an end point of the arc passing through $T$, or the arc $\mathcal{C}_{T}$ is wholly included in the two sides of $T$ having $v$ for ends.

Proof. Denote by $a$ and $b$ the ends of the arc passing through $T, \mathcal{C}_{T}$. From Proposition $7, \kappa\left(\mathcal{C}_{T}\right) \leq \frac{\pi}{2}$. Assume that $p \in \mathcal{C}_{T} \backslash\{a, b\}$ is a vertex of $T$. Then the geometric angle $\widehat{a p b}$ is less than or equal to $\frac{\pi}{2}$. Actually, it is equal to $\pi / 2$ for $\pi / 2 \geq \kappa\left(\mathcal{C}_{T}\right) \geq \pi-\widehat{a p b}$. Then, on the one hand, $a$ and $b$ lie on two adjacent edges of $T$ that intersect in $v$. On the other hand, we have $\kappa\left(\mathcal{C}_{T}\right)=\kappa\left(\mathcal{C}_{a}^{b}\right)=\kappa([a, p, b])$. Let $c$ be point in $\mathcal{C}$ in between $a$ and $p$. From the very definition of the turn, we derive that $\kappa([a, c, p, b])=\kappa([a, p, b])$, that is $c \in[a, p]$. Alike, any point of $\mathcal{C}$ in between $p$ and $b$ lie in the segment $[p, b]: \mathcal{C}_{T}$ is included in $[a, p] \cup[p, b]$.

Some point configurations cannot occur in the Gauss digitization of a curve compatible with the grid. Proposition 8 makes it possible to exclude some of these configurations. Indeed, we show that whether or not two 8-adjacent points in $h \mathbb{Z}^{2}$ are in the same connected component of $\mathbb{R}^{2} \backslash \mathcal{C}$ can be locally decided by considering the $\operatorname{arc} \mathcal{C}_{T}$ passing through a unit square $T$ having these points as vertices. Better, knowing the edges of $T$ on which lie the ends of $\mathcal{C}_{T}$ is sufficient to make the decision. Hence, instead of considering infinitely many cases (number of all possible LTB curves separating or not two 8-adjacent points), we only have to consider finitely many cases (i.e. all possible positions of the ends of the arc passing through $T$ ).

Proposition 8. Let $\mathcal{C}$ be $(\theta, \delta)-L T B$ Jordan curve, $T$ be a square compatible with the curve and $a, b$ be the end points of the arc passing through $T$. Two vertices of $T$ are in the same connected component of $\mathbb{R}^{2} \backslash \mathcal{C}$ if and only if they are in the same connected component of $T \backslash[a, b]$ and they do not lie on $\mathcal{C}$. 
Proof. Let consider the curve $\mathcal{C}^{\prime}=\left(\mathcal{C} \backslash \mathcal{C}_{T}\right) \cup[a, b]$ and the compact set $K$ delimited by the closed (non necessarily simple) curve $\mathcal{C}_{T} \cup[a, b]$. The proof is divided in three steps. In the first step, we prove that $\mathcal{C}^{\prime}$ is a Jordan curve. In the second step, we prove that if a vertex of $T$ is in $K$, then this vertex is on $\mathcal{C}$. In the third step, we prove that if two vertices of $T$ are in the same connected component of $\mathbb{R}^{2} \backslash \mathcal{C}$, then they are in the same connected component of $T \backslash[a, b]$.

- Step 1. The set $\mathcal{C}^{\prime}$ is a Jordan curve for $a$ and $b$ are the end points of $\mathcal{C} \backslash \mathcal{C}_{T}$ and $\mathcal{C} \backslash \mathcal{C}_{T}$ does not intersect $T$ (Proposition 7 ) while the segment $[a, b]$ is included in $T$.

- Step 2. By Propositions 6 and $7, \mathcal{C}_{T}$ is included in $T_{\pi / 2}$, the $\pi / 2$-swollen set of $T$. In particular, the vertices of $T$ lying in the compact set $K$, if any, belong to $\mathcal{C}_{T}$.

- Step 3. Two vertices of $T$ are in the same connected component of $\mathbb{R}^{2} \backslash \mathcal{C}$ if and only if they are in the same connected component of $\mathbb{R}^{2} \backslash \mathcal{C}^{\prime}$ and they do not lie in $\mathcal{C}$ (for, from Step 2., we know that they cannot lie in the interior of $K$ ), or, equivalently (since $\mathcal{C} \backslash \mathcal{C}_{T}$ does not intersect $T$ ), they are in the same component of $T \backslash[a, b]$ and they do not lie on $\mathcal{C}$.

We shall now prove that the Gauss digitization of a LTB curve is wellcomposed provided the grid step is small enough.

Proposition 9. Let $\mathcal{C}$ be $(\theta, \delta)$-LTB curve with $\theta \leq \pi / 2$ and $h$ be a grid step compatible with $\mathcal{C}$. Then, the Gauss digitization of $\mathcal{C}$ for the grid step $h$ is well-composed.

Proof. The proof is made by contradiction. So, let $a$ be a double point on $\partial_{h}(\mathcal{C})$. The point $a$ is the center of a square $T:=\left[I_{1}, E_{1}, I_{2}, E_{2}\right]$ whose vertices are points of $(h \mathbb{Z})^{2}$, the points $E_{1}$ and $E_{2}$ lying outside $\mathcal{C}$ while $I_{1}, I_{2}$ lie inside or on $\mathcal{C}$. Then, by discriminating vertices strictly inside $\mathcal{C}$ of vertices in $\mathcal{C}$, there are only three possible configurations modulo rotations and symmetries depicted in Figure 13. Let $\mathcal{C}_{p}^{q}$ be the arc passing through $T$.

- First configuration. By Proposition 8 , the segment $[p, q]$ separates the square $T$ into two polylines, the first containing the vertices $E_{1}$ and $E_{2}$ (outside $\mathcal{C}$ ) and the second (possibly empty) containing the vertices $I_{1}$ and $I_{2}$ (inside $\mathcal{C}$ ). The reader can check that this separating property does not hold for the first configuration of Figure 13.

- Second configuration. In the one hand, by Lemma $4, p$ or $q$ lies in the open polyline $\left(E_{1}, I_{1}, E_{2}\right)$ and, in the other hand, from Proposition $8, p$ and $q$ lie in the open polyline $\left(E_{1}, I_{2}, E_{2}\right)$.

- Third configuration. From Lemma $4,\{a, b\}=\left\{I_{1}, I_{2}\right\}$. Thus, the segment $\left[I_{1}, I_{2}\right]$ separates $E_{1}$ from $E_{2}$ in contradiction with Proposition 8. 

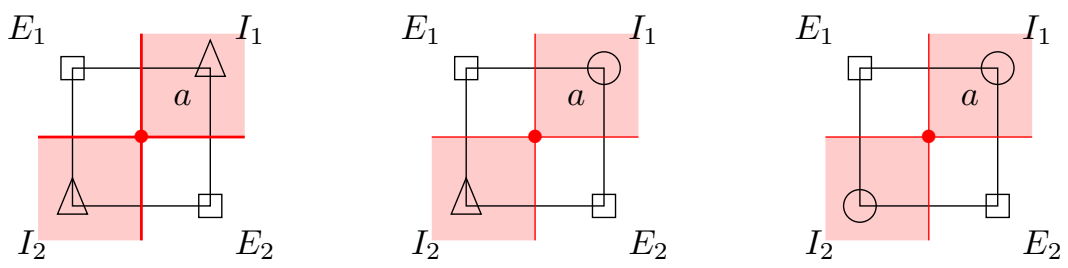

$-\partial_{h}(\mathcal{C}) \quad \triangle$ point inside $\mathcal{C} \square$ point outside $\mathcal{C} \bigcirc$ point on $\mathcal{C}$

Figure 13: The three possible configurations for a double point $a$ of the Gauss digitization of a Jordan curve.

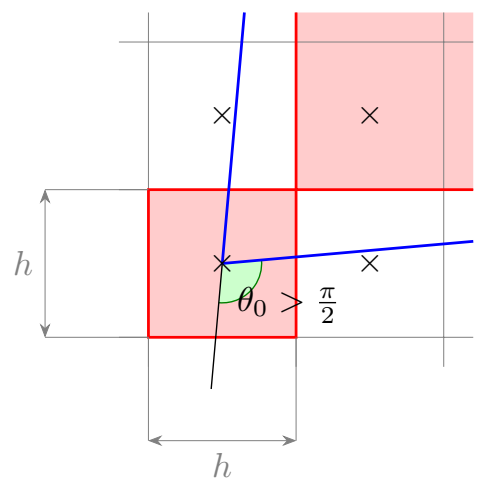

Figure 14: The blue spike with vertex at the origin is locally turn-bounded for any $\theta \geq \theta_{0}$ and any $\delta>0$. Nevertheless, its digitization is not well-composed (whatever the grid step).

Hence, none of the three configurations can occur.

Notice that the bounds $\theta \leq \frac{\pi}{2}$ and $\sqrt{2} h<\delta$ are tight: see Figure 14 and Figure 15 for counterexamples.

Corollary 5. Let $\mathcal{C}$ be $(\theta, \delta)-L T B$ curve with $\theta \leq \pi / 2$ and $h$ be a grid step compatible with $\mathcal{C}$. Then, the Gauss digitization of the closure of the interior of $\mathcal{C}$ is 4-connected.

Proof. Figure 16 illustrates the proof. Let $\mathcal{C}$ be a Jordan curve bounding a shape $S$ and $h>0$. Let $D$ be a connected component of the digitization of $\mathcal{C}$ (specifically, $D$ is the border of a connected component of the digitization of the shape $S$ ). Making a dilation of $D$ by the structuring element $h[-1 / 2,1 / 2] \times h[-1 / 2,1 / 2]$ centered in $(0,0)$ yields a new polygonal border $D^{\prime}$ whose vertices are integer points and edges are grid line segments. By the definition of $D$ and well-composedness (Proposition 9), no grid point in $D^{\prime}$ belongs to the digitization of the shape $S$. 


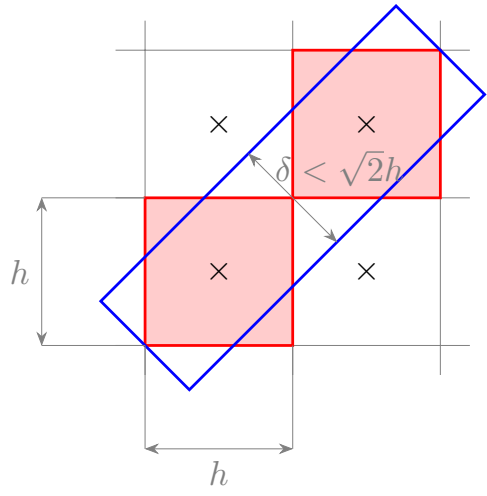

Figure 15: The digitization of the blue rectangle is not well-composed though its boundary is $(\pi / 2, \delta)$-locally turn-bounded.

Consider the collection $\mathcal{T}$ of all those unit squares sharing edges with $D^{\prime}$ : $\mathcal{T}=\left\{T_{i} \mid 1 \leq i \leq N_{D}\right\}$. Each unit square $T_{i}$ has at least a vertex outside $\mathcal{C}$. Moreover, by Proposition 9, there are exactly two edges of $T_{i}$ joining a vertex outside $\mathcal{C}$ to a vertex inside $\mathcal{C}$ or in $\mathcal{C}$. We claim that on each of these edges, there is an end of $\mathcal{C}_{T_{i}}$, the arc through $T_{i}$. Indeed, if there was not an end on an edge of $T_{i}$ joining a vertex $v_{e}$ outside $\mathcal{C}$ and a vertex inside $v_{e} \mathcal{C}$, by Proposition 8 , these two vertices would be in the same connected component of $\mathbb{R}^{2} \backslash \mathcal{C}$. And if there was not an end on an edge of $T_{i}$ joining a vertex $v_{e}$ outside $\mathcal{C}$ and a vertex $v_{b}$ on $\mathcal{C}$, by Lemma 4 , either the vertex $v_{b}$ is an end of $\mathcal{C}_{T_{i}}$, either $\mathcal{C}_{T_{i}}$ is wholly included in the two edges of $T_{i}$ having $v_{b}$ for edges, and $\mathcal{C}_{T_{i}}$ has one end on $\left[v_{e}, v_{b}\right]$.

Let $T_{1}$ and $T_{2}$ be two elements of $\mathcal{T}$ sharing an edge joining inside vertices to outside vertices. This edge contains an end point of the arc passing through $T_{1}$, denoted by $p_{1}$, and an end point of the arc passing through $T_{2}$, denoted by $p_{2}$. By Definition $8, p_{1}$ belongs to $\mathcal{C}_{T_{2}}$ and $p_{2}$ belongs to $\mathcal{C}_{T_{1}}$. Then, $\mathcal{C}_{T_{1}} \cup \mathcal{C}_{T_{2}}$ is an arc of $\mathcal{C}$ whose ends are respectively the ends of $\mathcal{C}_{T_{1}}$ and $\mathcal{C}_{T_{2}}$ distinct from $p_{1}$ and $p_{2}$. Eventually, going through $\mathcal{T}$, we build a closed arc $\bigcup_{i=1}^{N_{D}} C_{T_{i}}$ included in $\mathcal{C}$ and in the swollen set of $\bigcup \mathcal{T}$. As $\mathcal{C}$ is a Jordan curve, we derive that $\mathcal{C}=\bigcup_{i=1}^{N_{D}} C_{T_{i}}: D$ is unique.

Eventually, thanks to Proposition 9 and Corollary 5, we can now state the result announced at the beginning of this section.

Theorem 1. Let $\mathcal{C}$ be $(\theta, \delta)-L T B$ curve with $\theta \leq \pi / 2$ and $h$ be a grid step compatible with $\mathcal{C}$. Then, the Gauss digitization of $\mathcal{C}$ for the grid step $h$ is a Jordan curve whose interior is 4-connected.

In this section, we have proved that the hypothesis of local turn-boundedness guarantees the well-composedness for a small enough grid step. The well- 

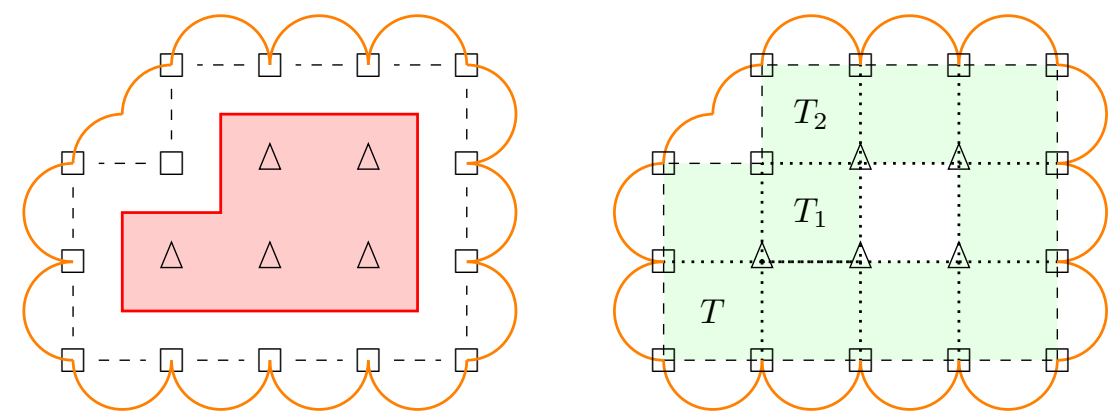

Figure 16: Proof of Corollary 5 (see text). Triangles: integer points of a connected component of the Gauss digitization of the shape $S$. Squares: integer points outside this component. Red thick line: the Gauss digitization $D$ of the curve $\mathcal{C}$. Dashed thin line: the border $D^{\prime}$ of the dilation of the Gauss digitization. Orange thick line: the border of $U$, the swollen set of $D^{\prime}$. Green: the collection of squares $\mathcal{T}$.

composedness of the digitization is also obtained under the hypothesis of parregularity. In the next section, we will show that local turn-boundedness is a relaxation of the par-regularity.

\section{$5 \quad$ Par-regularity and local turns}

Let us first give the statement of the main result of this section. Afterwards, we will recall the definition of par-regularity and give the outline of the proof.

Theorem 2. Let $\mathcal{C}$ be a par $(r)$-regular curve of class $\mathrm{C}^{1}$ and $\theta \in(0, \pi)$. Then, $\mathcal{C}$ is $(\theta, 2 r \sin (\theta / 2))-L T B$.

To introduce the notion of regularity, we use the same definition as in [7] and [8].

Definition 10 ( $\operatorname{par}(r)$-regularity). Let $\mathcal{C}$ be a Jordan curve of interior K.

- A closed ball $\bar{B}\left(c_{i}, r\right)$ is an inside osculating ball of radius $r$ to $\mathcal{C}$ at point $a \in \mathcal{C}$ if $\mathcal{C} \cap \bar{B}\left(c_{i}, r\right)=\{a\}$ and $\bar{B}\left(c_{i}, r\right) \subset K \cup\{a\}$.

- A closed ball $\bar{B}\left(c_{e}, r\right)$ is an outside osculating ball of radius $r$ to $\mathcal{C}$ at point $a \in \mathcal{C}$ if $\mathcal{C} \cap \bar{B}\left(c_{e}, r\right)=\{a\}$ and $\bar{B}\left(c_{e}, r\right) \subset \mathbb{R}^{2} \backslash(\mathcal{C} \cup K \cup\{a\}$.

- A curve $\mathcal{C}$ or a set $K$ is par(r)-regular if there exist inside and outside osculating balls of radius $r$ at each $a \in \mathcal{C}$.

The proof of Theorem 2 is divided into three steps. The first two steps are independent. In the first step, we show that the turn of a par $(r)$-regular curve is a $\frac{1}{r}$-Lipschitz function of its length (Lemma 5 ). In the second step, we show that the distance between the ends of a small arc of a $\operatorname{par}(r)$-regular curve is an 


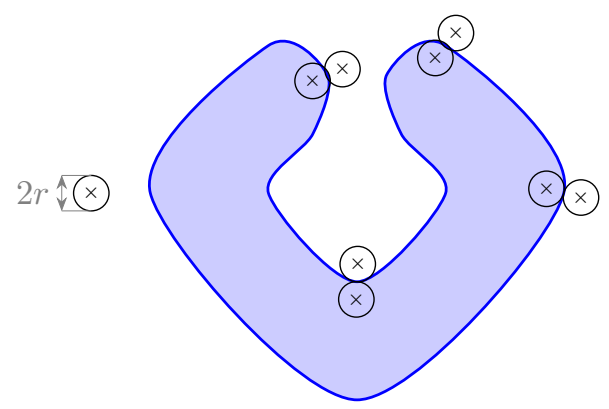

Figure 17: The par( $r)$-regularity demands that at each point of the boundary of the shape, there exist inside and outside osculating balls of radius $r$.

increasing function of its length (Lemma 6). In the last step, applying Schur's Comparison Theorem to a $\operatorname{par}(r)$-regular arc of length $\theta r$ and a circle arc of radius $r$ and turn $\theta$, we show that the distance between the end points of the $\operatorname{par}(r)$-regular arc is greater than $2 r \sin \left(\frac{\theta}{2}\right)$. Since this distance is an increasing function of the length (Lemma 6), we derive that the length of the $\operatorname{par}(r)$-regular arc between points at distance $2 r \sin \left(\frac{\theta}{2}\right)$ is smaller than $\theta r$ (Proposition 11). Then, thanks to Lemma 5 - the turn of a $\operatorname{par}(r)$-regular curve is a $\frac{1}{r}$-Lipschitz function of its length - we conclude the proof.

Step 1: the turn of a $\operatorname{par}(r)$-regular arc is a $\frac{1}{r}$-Lipschitz function of its length.

Some elementary lemmae used in this paragraph are stated in Appendix E .

The following lemma shows that the turn of a $\operatorname{par}(r)$-regular curve is a $\frac{1}{r}$ Lipschitz function of the length.

Lemma 5. Let $\mathcal{C}$ be a par(r)-regular curve. Then the length of any arc $\mathcal{A}$ of $\mathcal{C}$ is greater than, or equal to the length of a circle arc with radius $r$ and turn $\kappa(\mathcal{A})$. In other words, for each arc $\mathcal{A}$ of $\mathcal{C}$,

$$
r \kappa(\mathcal{A}) \leq \mathcal{L}(\mathcal{A}) .
$$

Proof. We denote by $a$ and $b$ the endpoints of the $\operatorname{arc} \mathcal{A}$. For each $m \in \mathbb{N}^{*}$, let $\left(a_{m, i}\right)_{i \in \llbracket 0, N_{m} \rrbracket}$ be the ordered sequence of vertices of a polygonal line $L_{m}$ inscribed in $\mathcal{A}$ such that $a_{m, 0}=a, a_{m, N_{m}}=b$ and

$$
\forall i \in \llbracket 0, N_{m}-2 \rrbracket,\left\|a_{m, i+1}-a_{m, i}\right\|=\frac{1}{m}<2 r .
$$

and $\left\|a_{m, N_{m}}-a_{m, N_{m}-1}\right\| \leq \frac{1}{m}$. Then, from Lemma 9 (Appendix E) and since 
the function arcsine is increasing,

$$
\begin{aligned}
\kappa\left(L_{m}\right) & \leq 2\left(\left\lceil\frac{\mathcal{L}\left(L_{m}\right)}{1 / m}\right\rceil-1\right) \arcsin \left(\frac{1 / m}{2 r}\right), \\
& \leq 2 \frac{\mathcal{L}\left(L_{m}\right)}{1 / m} \arcsin \left(\frac{1 / m}{2 r}\right) .
\end{aligned}
$$

Moreover, by Property 1,

$$
\begin{aligned}
\lim _{1 / m \rightarrow 0} \mathcal{L}\left(L_{m}\right) & =\mathcal{L}(\mathcal{A}) \text { and }, \\
\lim _{1 / m \rightarrow 0} \kappa\left(L_{m}\right) & =\kappa(\mathcal{A}) .
\end{aligned}
$$

Furthermore,

$$
\lim _{1 / m \rightarrow 0} \frac{2 r}{1 / m} \arcsin \left(\frac{1 / m}{2 r}\right)=1
$$

Hence

$$
\kappa(\mathcal{A}) \leq \frac{1}{r} \mathcal{L}(\mathcal{A}) .
$$

\section{Step 2: Par-regular curves have a local quasiconvex behavior.}

This step uses the derivative of a par-regular curve. This is possible because par-regularity was indirectly proven to imply continuous differentiability. Indeed, in [7], Lachaud and Thibert show that par-regularity is equivalent to having positive reach which was proven by Federer [4] to be equivalent to being of class $\mathrm{C}^{1,1}$ ( $\mathrm{C}^{1}$ with Lipschitz derivative). We give below a proof based on the work of Alexandrov and Reshetnyak [1].

Proposition 10. Every par( $(r)$-regular curve $\mathcal{C}$ is of class $\mathrm{C}^{1}$.

Proof. Let $a \in \mathcal{C}$ and $B_{i}, B_{e}$ be respectively the interior and exterior osculating balls of radius $r$ at $a$. Let $D$ be the common tangent to $B_{i}$ and $B_{e}$ at $a$. Since $\mathcal{C} \backslash\{a\}$ does not intersect $B_{i}$ and $B_{e}$, it is easy to see that, for any $\epsilon>0$ and any point $b$ in the curve neighborhood $\mathcal{C} \cap B(a, 2 r \cos (\epsilon))$, the angle between the straight line $a b$ and $D$ is less than $\epsilon$. Also, observe that the definition of parregularity forbids cusps. Then, by Definition $2, \mathcal{C}$ has left-hand and right-hand tangents in $a$ which are equal: $\mathcal{C}$ is a smooth curve whose tangents everywhere coincide with those of its osculating balls. Eventually, we derive from Property 5 that $\mathcal{C}$ is of class $\mathrm{C}^{1}$.

The following lemma states that, for any injective parametrization $\gamma$ of a par-regular curve $\mathcal{C}$, the distance function $t \mapsto\left\|\gamma(t)-\gamma\left(t_{0}\right)\right\|$ is quasiconvex near its minimum. 
Lemma 6. Let $\mathcal{C}$ be a par(r)-regular curve and a a point on $\mathcal{C}$. Let $\mathcal{A}$ be the intersection of $\mathcal{C}$ with the ball $B(a, 2 r)$. Then, $\mathcal{A}$ is path-connected and for any injective parametrization $\gamma$ the distance function $t \mapsto\|\gamma(t)-a\|$ is quasiconvex.

Proof. Let $\gamma$ be an injective parametrization of $\mathcal{C}$. By contradiction, assume that there exists a local minimum $c \neq \gamma^{-1}(a)$ of the map $\phi: t \mapsto\|\gamma(t)-a\|$. By Proposition $10, \mathcal{C}$ is of class $\mathrm{C}^{1}$, then, $\phi^{\prime}(c)=0$. Hence

$$
<\gamma^{\prime}(c), \gamma(c)-a>=0,
$$

that is, $\gamma^{\prime}(c)$ is orthogonal to $\gamma(c)-a$. The osculating disks of radius $r$ at $\gamma(c)$ are tangent to $\gamma^{\prime}(c)$ and $\|\gamma(c)-a\| \leq r$. It follows that the point $a$ is in one of the osculating disks at $\gamma(c)$ which contradicts the assumption of $\operatorname{par}(r)$-regularity. Moreover assume that $\mathcal{A}$ is not path-connected. Let $\mathcal{C}_{1}$ be a connected component which is not containing $a$. By Rolle's Theorem, there exists $t_{0}$ such that $\phi^{\prime}\left(t_{0}\right)=0$ and $\gamma\left(t_{0}\right) \in B(a, 2 r)$, which is impossible.

We can prove an equivalent statement of Lemma 6 for LTB curves; See Appendix C. These similar behaviors are not surprising since we are showing that par-regularity implies local turn boundedness. Nevertheless, it is interesting to compare the radii of the neighborhoods in which these local properties hold: $2 r$ in the one hand (par $(r)$-regularity), $\delta$ in the other hand $((\theta, \delta)$-local turn boundedness) while Theorem 2 states that $\operatorname{par}(r)$-regularity implies $(\theta, 2 r \sin (\theta / 2))$ local turn boundedness where $\theta \in(0, \pi)$. Then, the radii coincide in the limit case $\theta=\pi$.

\section{Step 3: Applying Schur's Comparison Theorem}

Proposition 11. Let $\mathcal{C}$ be a par(r)-regular curve and $\theta \in[0, \pi)$. Given two points $a, b$ in $\mathcal{C}$ such that $\|b-a\| \leq 2 r \sin \left(\frac{\theta}{2}\right)$, the arc of $\mathcal{C}$ joining $a$ to $b$ in $B(a, 2 r)$ has its length smaller than or equal to $\theta r$.

Proof. Let $\gamma$ be the parametrization by arc length of the arc of $\mathcal{C}$ from $a$ to $b$ in $B(a, 2 r)$. Then, $\gamma(0)=a$ and $\gamma\left(s_{1}\right)=b$ for some $s_{1}>0$. By contradiction, assume that $s_{1}>\theta r$ and put $c=\gamma(\theta r)$.

Let $\bar{\gamma}$ be the parametrization by arc length of some circle of radius $r$.

By Lemma 5, for any subinterval $I$ of $[0, \theta r]$,

$$
\kappa(\gamma(I)) \leq \frac{1}{r}|I| .
$$

In other words, for any subinterval $I$ of $[0, \theta r]$,

$$
\kappa(\gamma(I)) \leq \kappa(\bar{\gamma}(I)) .
$$

Hence, Schur's Comparison Theorem applies:

$$
\begin{aligned}
\|c-a\| & \geq\|\bar{\gamma}(\theta r)-\bar{\gamma}(0)\| \\
& \geq 2 r \sin \left(\frac{\theta}{2}\right) \\
& \geq\|b-a\| .
\end{aligned}
$$


The last inequality contradicts the quasi-convexity of $s \mapsto\|\gamma(s)-\gamma(0)\|$ (Lemma 6).

Theorem 2. Let $\mathcal{C}$ be a par( $(r)$-regular curve of class $\mathrm{C}^{1}$ and $\theta \in(0, \pi)$. Then, $\mathcal{C}$ is $(\theta, 2 r \sin (\theta / 2))$-locally turn-bounded.

Proof. By Proposition 11, the length of one of the arc of $\mathcal{C}$ delimited by two points at distance less than $2 r \sin \left(\frac{\theta}{2}\right)$ is at most $\theta r$. Hence by Lemma 5 the turn of one of the arc of $\mathcal{C}$ delimited by two points at distance less than $2 r \sin \left(\frac{\theta}{2}\right)$ is at most $\theta$.

Notice that the circle is not $(\theta, \delta)$-LTB for $\delta$ greater than $2 r \sin \left(\frac{\theta}{2}\right)$, hence the value of $\delta$ given in Theorem 2 is optimal.

Let us now compare our condition for well-composedness of $(\theta, \delta)$-LTB curves with respect to the grid step $h, \sqrt{2} h<\delta$ with $\theta \leq \pi / 2$ (Definition 9), with the condition of Pavlidis [13, Definition 7.4] for $\operatorname{par}(r)$-regular curves, $\sqrt{2} h<2 r$. Using Theorem 2, the assumption $\sqrt{2} h<\delta$ applied on a $\operatorname{par}(r)$-regular curve becomes

$$
\sqrt{2} h<2 r \sin \left(\frac{\theta}{2}\right) \text {. }
$$

Hence our compatibility hypothesis, which also applies to non-smooth curves, requires a smaller grid step when applied on smooth curves (for $\theta=\frac{\pi}{2}, \sqrt{2}$ times smaller).

\section{Conclusion}

In this paper, the notion of local turn boundedness, which is adapted to both regular curves and polygons having large enough interior angles, was developed to have control on curves without smoothness assumption.

The LTB curves are a subset of curves of finite length and finite turn. They have been designed to exclude curves for which geometric estimation is not possible: they cannot have small oscillations and the distance to their digitization is bounded. They have their intrinsic properties: they are locally connected, they cannot do small U-turns.

From these intrinsic properties, we have derived some properties of their digitization. In particular, we were able to precisely describe their behavior when passing through sufficiently small pixels and how they separate grid points. Then, topological properties as the well-composedness and 4-connectedness of the curve Gauss digitization was deduced. Finally, local turn boundedness was proven to generalize par-regularity. Since par-regularity amounts to having positive reach [7] and since the reach was relaxed by the notion of $\mu$-reach for use with non smooth curves, we recently began to compare the $\mu$-reach [3] with the local turn boundedness and we hope to be able to present soon some results about this comparison.

In a future work, using the results of this article, we intend to define maps associating sampling points of a Gauss digitization to near points on the continuous curve without smoothness assumption. Moreover, the definition of local-turn 
seems to generalize without change to curves and surfaces in a three dimensional space. Nevertheless, some properties like well-composedness cannot be extended to the 3-dimensional case (see counterexample in [15, Figure 4]) and the extension of other properties has to be proven. In the long term, we hope that local turn will provide a framework more general than the par-regularity, for both geometric estimation and topology preservation.

\section{A Proof of Corollary 1}

Lemma 7. The boundary of a convex shape with nonempty interior has no cusp.

Proof. Let $\mathcal{C}$ be a Jordan curve whose interior is convex. Let $p \in \mathcal{C}$. Since $\mathcal{C}$ is a Jordan curve, there exist in $\mathcal{C}$ two points $q, r$ not colinear with $p$. Let $P, Q, R$ be three straight lines passing respectively by the points $p, q, r$ and separating $\mathcal{C}$ from one of the half planes they delimit (see Figure 18). The interior of the convex hull of $\{p, q, r\}$ is included in the interior of $\mathcal{C}$. Then the arc from $q$ to $r$ passing through $p$ is included in the half plane delimited by $P$ and containing $p q r$ deprived of the triangle $p q r$. Then, if $\mathcal{C}$ has demi-tangent vectors $\mathbf{u}$ and $\mathbf{v}$ in $p$, the angle $\angle(\mathbf{u}, \mathbf{v})$ is bounded from above by $\pi-\alpha$ where $\alpha$ is the interior angle of the triangle $[p, q, r]$ at $p$.

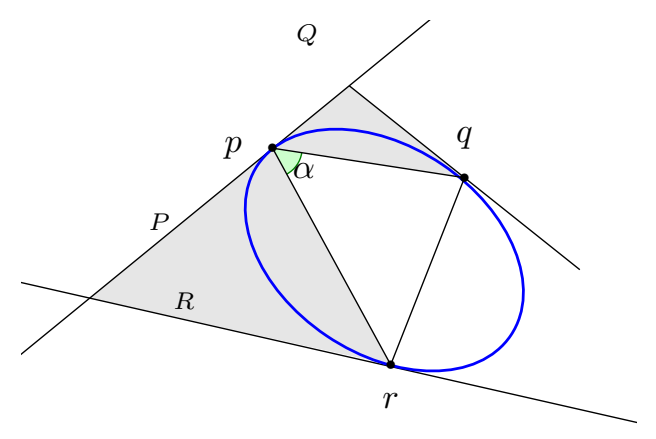

Figure 18: Bounding a convex curve (in blue) passing through three non colinear points.

\section{B Proof of Lemma 1}

Lemma 1. Let $\mathcal{C}$ be a $(\theta, \delta)-L T B$ curve with $\theta<2 \pi / 3$. Then,

$$
\delta \leq \operatorname{diam}(\mathcal{C})
$$

where diam denotes the diameter. 
Proof. By contradiction, we assume $\delta>\operatorname{diam}(\mathcal{C})$. Let $\ell$ be the length of $\mathcal{C}, a$ be a non-angular point of $\mathcal{C}$ and $k<1 / 2$. We prove by induction that, for any $n$, there exists an $\operatorname{arc} \mathcal{C}_{n}$ of $\mathcal{C}$ whose ends are non-angular points and containing $a$ whose turn is less than $\theta$ and whose length is greater than $\left(1-(1-k)^{n}\right) \ell$. Furthermore, the sequence $\left(\mathcal{C}_{i}\right)$ is increasing for the inclusion. We initialize the induction by taking a smooth point $b$ of $\mathcal{C}$ such that the geodesic distance from $a$ to $b$ is greater than $k \ell$ (recall that the set of angular points of a LTB-curve is countable). Since $\delta>\operatorname{diam}(\mathcal{C})$ and $\mathcal{C}$ is LTB, one of the arcs from $a$ to $b$, that we denote by $\mathcal{C}_{1}$, has a turn less than or equal to $\theta$. Assuming the property is true for some $i \geq 1$, we denote by $a_{i}$ and $b_{i}$ the end points of $\mathcal{C}_{i}$. There exists a smooth point $c \in \mathcal{C} \backslash \mathcal{C}_{i}$ such that the geodesic distance from $c$ to both $a_{i}$ and $b_{i}$ is greater than or equal to $k\left(\ell-\mathcal{L}\left(\mathcal{C}_{i}\right)\right)$. If the arc from $a_{i}$ to $c$ not passing through $b_{i}$ has a turn less than $\theta$, we set $a_{i+1}=c, b_{i+1}=b_{i}$ and $\mathcal{C}_{i+1}$ is the arc from $a_{i+1}$ to $b_{i+1}$ (that is from $c$ to $b_{i}$ ) passing through $a_{i}$. Indeed, the other arc from $a_{i+1}$ to $b_{i+1}$ has a turn greater than $2 \pi-2 \theta$ by Fenchel's Theorem (Property 3 ) and Property 7 . So, since $\mathcal{C}$ is $(\theta, \delta)$-LTB and $\mathrm{d}\left(a_{i+1}, b_{i+1}\right) \leq \operatorname{diam}(\mathcal{C})<\delta$, the turn of $\mathcal{C}_{i+1}$ is less than $\theta$. Moreover, $\mathcal{C}_{i} \subseteq \mathcal{C}_{i+1}$. If the arc from $a_{i}$ to $c$ not passing through $b_{i}$ has a turn greater than $\theta$, we define $\mathcal{C}_{i+1}$ as the arc from $a_{i}$ to $c$ passing through $b_{i}$ since it has a turn less than $\theta$ and we set $a_{i+1}=a_{i}, b_{i+1}=c$. In both cases, we have $\mathcal{C}_{i} \subseteq \mathcal{C}_{i+1}$ and $\mathcal{L}\left(\mathcal{C}_{i+1}\right) \geq \mathcal{L}\left(\mathcal{C}_{i}\right)+k\left(\ell-\mathcal{L}\left(\mathcal{C}_{i}\right)\right)$, and, since $\mathcal{L}\left(\mathcal{C}_{i}\right) \geq\left(1-(1-k)^{i}\right) \ell$ by induction hypothesis, we obtain $\mathcal{L}\left(\mathcal{C}_{i+1}\right) \geq\left(1-(1-k)^{i+1}\right) \ell$. This completes the induction. Now, on the one hand, considering the $\operatorname{arc} \mathcal{C}_{\infty}=\bigcup \mathcal{C}_{i}$, we claim that $\mathcal{C}_{\infty}$ has a length greater than $\left(1-(1-k)^{i}\right) \ell$ for any positive integer $i$. Thus, $\mathcal{L}\left(\mathcal{C}_{\infty}\right)=\ell$. Then, $\mathcal{C} \backslash \mathcal{C}_{\infty}$ is reduced to a point. On the other hand, since $\mathcal{C}_{\infty}$ is the supremum of an increasing sequence of arcs whose turns are bounded from above by $\theta$, it also has a turn bounded from above by $\theta$. This contradicts Corollary 1.

\section{Local increase of the distance for $(\theta, \delta)$-LTB curves}

We now show that, when $\theta \leq \pi / 2$, the Euclidean distance $d(p, q)$ between two points $p$ and $q$ of a parameterized LTB curve is locally monotonic in function of the parameter of one of the two points $p$ and $q$. Visually, Proposition 12 states that $(\theta, \delta)$-LTB have no local U-turns (see Figure 19).

Proposition 12. Let $\theta \in(0, \pi / 2]$ and $\mathcal{C}$ be $a(\theta, \delta)-L T B$ curve . Let $\gamma:\left[0, t_{M}\right] \rightarrow$ $\mathcal{C}$ be an injective parametrization of the curve $\mathcal{C}$ and $t_{m} \in\left(0, t_{M}\right)$ be such that the arc $\gamma\left(\left[0, t_{m}\right]\right)$ is included in $B\left(\gamma(0), \frac{\delta}{2}\right)$. Then, the restriction of the function $t \mapsto\|\gamma(t)-\gamma(0)\|$ on $\left[0, t_{m}\right]$ is increasing.

Proof. We prove a contrapositive statement. Let $\phi: t \in\left[0, t_{m}\right] \mapsto \| \gamma(t)-$ $\gamma(0) \|$. Suppose that $\phi$ is not monotonic. Then, there exists $t_{1}, t_{2}$ in $\left(0, t_{m}\right)$ 


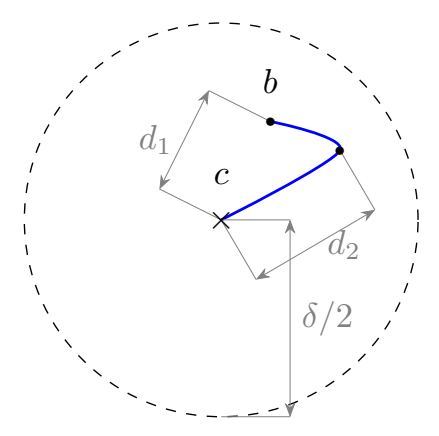

Figure 19: This configuration cannot occur in a $(\theta, \delta)$-LTB since the distance to the point $c$ is not locally increasing $\left(d_{1}<d_{2}\right)$.

such that $t_{1}<t_{2}$ and $\phi\left(t_{1}\right)>\phi\left(t_{2}\right)$. Therefore, the turn of the polygonal line $\left[\gamma(0), \gamma\left(t_{1}\right), \gamma\left(t_{2}\right)\right]$ is strictly greater than $\pi / 2$. Hence, the turn of the arc $\gamma\left(\left[0, t_{2}\right]\right)$ is a fortiori greater than $\pi / 2$. Since $\mathcal{C}$ is $(\theta, \delta)$-LTB for some $\theta<$ $\pi / 2$, the turn of the arc $\gamma\left(\left[t_{2}, t_{M}\right]\right)$ is strictly less than $\pi / 2$ and, according to Proposition 4 , the arc $\gamma\left(\left[t_{2}, t_{M}\right]\right)$ is therefore included in the disk of diameter $\left[\gamma\left(t_{2}\right), \gamma\left(t_{M}\right)\right]$ which is itself included in the ball $B(\gamma(0), \delta / 2)\left(\right.$ for $\left.\gamma\left(t_{M}\right)=\gamma(0)\right)$. We conclude that the whole curve $\mathcal{C}$ is included in the ball $B\left(\gamma(0), \frac{\delta}{2}\right)$. Then the diameter of $\mathcal{C}$ is strictly less than $\delta$ which contradicts Lemma 1.

\section{Proof of Proposition 7}

Proposition 7. Let $\theta \in(0, \pi / 2]$ and $\mathcal{C}$ be $a(\theta, \delta)-L T B$ Jordan curve. Let $d$ be the diameter of $\mathcal{C}$. Let $T$ be a closed set included in an open disk $B(c, r)$ with $r$ less than or equal to $\min \left(\frac{1}{2} \delta, \frac{\sqrt{2}}{4} d\right)$. Then, the arc $\mathcal{C}_{T}$ passing through $T$ is the unique arc of $\mathcal{C}$ of turn less than or equal to $\frac{\pi}{2}$ having its end points in $T$ and such that the straightest arc between any two points of $T$ is included in $\mathcal{C}_{T}$. Moreover,

$$
\left(\mathcal{C} \backslash \mathcal{C}_{T}\right) \cap T=\emptyset .
$$

Proof. Observe that, obviously, any straightest arc between two points of $\mathcal{C} \cap T$ is included in $\mathcal{C}_{T}$. Furthermore,

$$
\mathcal{C} \cap T=\bigcup_{a \in \mathcal{C} \cap T} \mathcal{C}_{a}^{a} \subset \mathcal{C}_{T},
$$

where $\mathcal{C}_{a}^{a}=\{a\}$ is the straightest arc from $a$ to $a$. Thus,

$$
\left(\mathcal{C} \backslash \mathcal{C}_{T}\right) \cap T=\emptyset .
$$

The proof is divided into six steps. In the first step, we show that $\mathcal{C}_{T}$ is an arc of $\mathcal{C}$. In the second step, we show that the ends of $\mathcal{C}_{T}, q_{1}$ and $q_{2}$, are in $T$. 
In the third step, we show that $\mathcal{C}_{T}$ is the straightest arc between $q_{1}$ and $q_{2}$. In the fourth step, we show that $\mathcal{C}_{T}$ is included in the open disk $B(c, \sqrt{2} r)$ (see Figure 20). In the fifth step, we show that $\mathcal{C}_{T}$ is not the whole curve $\mathcal{C}$. In the sixth step, we show that $\mathcal{C}_{T}$ is the unique arc of $\mathcal{C}$ with turn less than or equal to $\pi / 2$ and including any straightest arc of $\mathcal{C}$ between points of $T$.

Step 1. Let $p_{1}$ and $p_{2}$ be two points of $\mathcal{C}_{T}$. By Definition 6 , there exist four points $a_{1}, b_{1}, a_{2}, b_{2}$ in $T$ such that $p_{1}$ lies in the straightest arc $\mathcal{C}_{a_{1}}^{b_{1}}$ and $p_{2}$ lies in the straightest $\operatorname{arc} \mathcal{C}_{a_{2}}^{b_{2}}$. Hence, the straightest arc between $a_{1}$ and $a_{2}$, which is included in $\mathcal{C}_{T}$, connects the arcs $\mathcal{C}_{a_{1}}^{b_{1}}$ and $\mathcal{C}_{a_{2}}^{b_{2}}$. Thereby, $p_{1}$ and $p_{2}$ are connected by a path in $\mathcal{C}_{T}$. We derive that $\mathcal{C}_{T}$ is path-connected: $\mathcal{C}_{T}$ is an arc of $\mathcal{C}$.

Step 2. The ends of $\mathcal{C}_{T}, q_{1}$ and $q_{2}$, are limits of points that are the ends of straightest arcs between two points in $T$. Indeed, for any $\epsilon>0$, there exists a point $q_{1}^{\prime}$ of $\mathcal{C}_{T}$ such that the length of the subarc of $\mathcal{C}_{T}, \mathcal{C}_{q_{1}}^{q_{1}^{\prime}}$, is less than $\epsilon$. The point $q_{1}^{\prime}$ belongs to a straightest arc between two points in $T$, one of these two points is on the $\operatorname{arc} \mathcal{C}_{q_{1}}^{q_{1}^{\prime}}$, hence at geodesic distance from $q_{1}$ less than $\epsilon$ and a fortiori at Euclidean distance from $q_{1}$ less than $\epsilon$. The same holds for $q_{2}$. Since $T$ is a closed set, $\mathcal{C}_{T}$ has its end points $q_{1}$ and $q_{2}$ in $T$.

Step 3. The straightest arc between $q_{1}$ and $q_{2}$ is included in $\mathcal{C}_{T}$ (by definition of $\mathcal{C}_{T}$ ). Then, $\mathcal{C}_{T}$ is the straightest arc between $q_{1}$ and $q_{2}$.

Step 4. (Figure 20) From Step 3 and Proposition 4, we derive that $\mathcal{C}_{T}$ is included in the disk of diameter $\left[q_{1}, q_{2}\right]$. By the hypotheses, the segment $\left[q_{1}, q_{2}\right]$ is included in $B(c, r)$. Then, the $\operatorname{arc} \mathcal{C}_{T}$ is included in the open ball $B\left(c,\left(\sin \frac{\phi}{2}+\cos \frac{\phi}{2}\right) r\right) \subseteq B(c, \sqrt{2} r)$ where $\phi$ is the geometric angle $\widehat{q_{1} c q_{2}}$.

Step 5. By hypothesis, the diameter of $\mathcal{C}$ is greater than or equal to $2 \sqrt{2} r$. Since $\mathcal{C}_{T}$ is included in the open disk $B(c, \sqrt{2} r), \mathcal{C}_{T}$ cannot be the whole curve $\mathcal{C}$.

Step 6. If there exits another $\operatorname{arc} \mathcal{C}^{\prime}$ of curvature less than or equal to $\frac{\pi}{2}$ having its ends in $T$ such that each other arc having its ends in $T$ and of turn less than or equal to $\frac{\pi}{2}$ is included in $\mathcal{C}^{\prime}$, then $\mathcal{C}^{\prime} \subset \mathcal{C}_{T}$ and $\mathcal{C}_{T} \subset \mathcal{C}^{\prime}$ hence $\mathcal{C}^{\prime}=\mathcal{C}_{T}$.

\section{E Turn of a polygonal line inscribed in a par- regular curve}

We establish two results allowing us to control the turn of a polygonal line inscribed in a $\operatorname{par}(r)$-regular curve (Lemma 8 and Lemma 9). The first of 


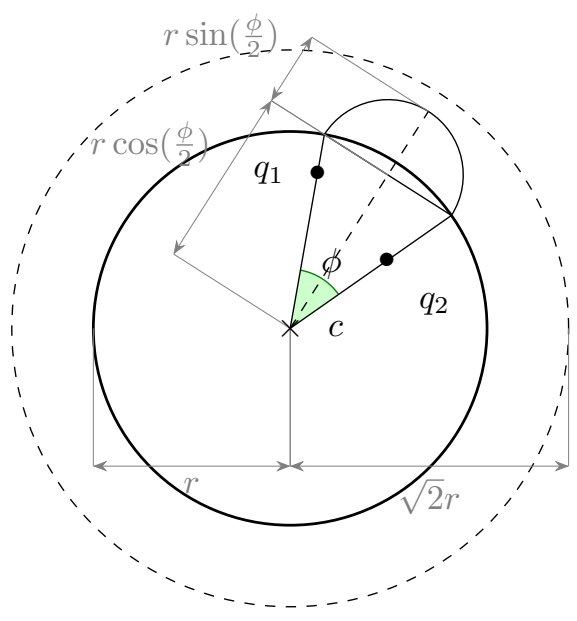

Figure 20: Step 4 of the proof of Proposition 7: the points $q_{1}$ and $q_{2}$ are in the ball of center $c$ and of radius $r$. Then the ball of diameter $\left[q_{1}, q_{2}\right]$ is included in the ball $B(c, \sqrt{2} r)$ for $\sin \frac{\phi}{2}+\cos \frac{\phi}{2} \leq \sqrt{2}$.

these two lemmas is an easy consequence of the inscribed angle theorem. It is illustrated in Figure 21. Its proof is left to the reader.

Lemma 8. Let $P$ be a polygonal line $\left[a_{i}\right]_{i=0}^{N}$. Then,

$$
\begin{aligned}
\kappa(P) & =\frac{1}{2} \sum_{i=1}^{N-1} \theta_{i} \\
& =\sum_{i=1}^{N-1} \arcsin \left(\frac{\left\|a_{i}-a_{i-1}\right\|}{2 r_{i}}\right) \\
& +\arcsin \left(\frac{\left\|a_{i+1}-a_{i}\right\|}{2 r_{i}}\right),
\end{aligned}
$$

where, for any $i \in \llbracket 1, N-1 \rrbracket, r_{i}$ is the radius of the circumcircle $C_{i}$ of the triangle $a_{i-1} a_{i} a_{i+1}$ and $\theta_{i}$ is the central angle of $C_{i}$ subtended by the arc $\left[a_{i-1}, a_{i}, a_{i+1}\right]$.

Lemma 9. Let $P=\left[a_{i}\right]_{i=0}^{N}$ be a polygonal line inscribed in a par( $\left.r\right)$-regular curve. If the maximal edge length of $P$ is less than $2 r$, then the turn of $P$ is less than the turn of a polygonal line which has the same edge length sequence and 


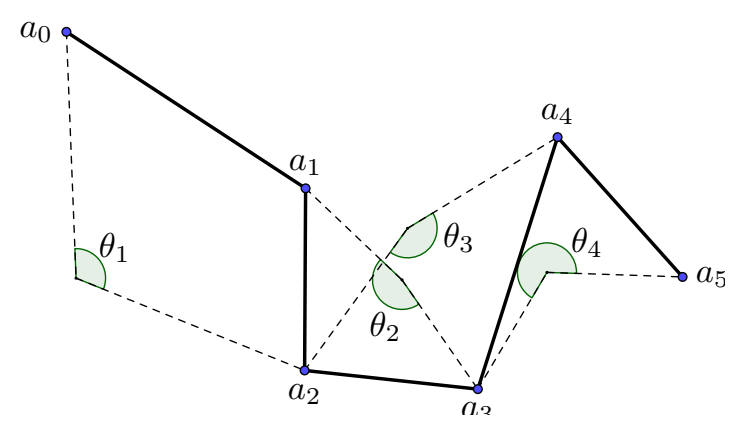

Figure 21: Thick: the polygonal line $P=\left[a_{0}, a_{1}, a_{2}, a_{3}, a_{4}, a_{5}\right]$. The four angles $\theta_{1} \theta_{2}, \theta_{3}$ and $\theta_{4}$ are subtended by the circle arcs defined respectively by the triples $\left(a_{0}, a_{1}, a_{2}\right),\left(a_{1}, a_{2}, a_{3}\right),\left(a_{2}, a_{3}, a_{4}\right)$ and $\left(a_{3}, a_{4}, a_{5}\right)$. Then, the turn of $P$ is half the sum of the $\theta_{i}: \kappa(P)=\frac{1}{2} \sum_{i=1}^{4} \theta_{i}$.

is inscribed in a circle of radius $r$. In other words,

$$
\begin{aligned}
\kappa(P) \leq & \arcsin \left(\frac{\left\|a_{1}-a_{0}\right\|}{2 r}\right) \\
& +2 \sum_{i=1}^{N-2} \arcsin \left(\frac{\left\|a_{i+1}-a_{i}\right\|}{2 r}\right) \\
& +\arcsin \left(\frac{\left\|a_{N}-a_{N-1}\right\|}{2 r}\right) .
\end{aligned}
$$

Proof. Figure 22 illustrates the main argument of the proof. Let $\mathcal{C}$ be a $\operatorname{par}(r)$ regular curve and $P=\left[a_{i}\right]_{i=0}^{N}$ be a polygonal line inscribed in $\mathcal{C}$. For any $i \in \llbracket 0, N-1 \rrbracket$, we set $\ell_{i}=\left\|a_{i+1}-a_{i}\right\|$ and we assume $\ell_{i} \leq 2 r$. Let $P^{\prime}=\left[a_{i}^{\prime}\right]_{i=0}^{N}$ be a polygonal line inscribed in a circle with radius $2 r$ such that $\ell_{i}=\left\|a_{i+1}^{\prime}-a_{i}^{\prime}\right\|$ for any $i \in \llbracket 0, N-1 \rrbracket$.

For each $i \in \llbracket 1, N-1 \rrbracket$, let us denote respectively by $\kappa_{i}$ and $\kappa_{i}^{\prime}$ the turn of the polygonal lines $\left[a_{i-1}, a_{i}, a_{i+1}\right]$ and $\left[a_{i-1}^{\prime}, a_{i}^{\prime}, a_{i+1}^{\prime}\right]$. On the one hand, from Lemma 8 (Appendix E, we have $\kappa_{i}^{\prime}=\arcsin \left(\frac{\ell_{i-1}}{2 r}\right)+\arcsin \left(\frac{\ell_{i+1}}{2 r}\right)$. On the other hand, the turns $\kappa\left(\left[a_{i-1}, a_{i}, a_{i+1}\right]\right)$ and $\kappa\left(\left[a_{i-1}^{\prime}, a_{i}^{\prime}, a_{i+1}^{\prime}\right]\right)$ are respectively the supplementary angles of $\widehat{a_{i-1} a_{i} a_{i+1}}$ and $\widehat{a_{i-1}^{\prime}} \widehat{a_{i}^{\prime} a_{i+1}^{\prime}}$. Then, from the definition 10 of the par-regularity, $\kappa\left(\left[a_{i-1}, a_{i}, a_{i+1}\right]\right) \leq \kappa\left(\left[a_{i-1}^{\prime}, a_{i}^{\prime}, a_{i+1}^{\prime}\right]\right)$ (see Figure 22). As $\kappa(P)=\sum_{i=1}^{N} \kappa_{i}$ and $\kappa\left(P^{\prime}\right)=\sum_{i=1}^{N} \kappa_{i}^{\prime}$, we conclude straightforwardly. 


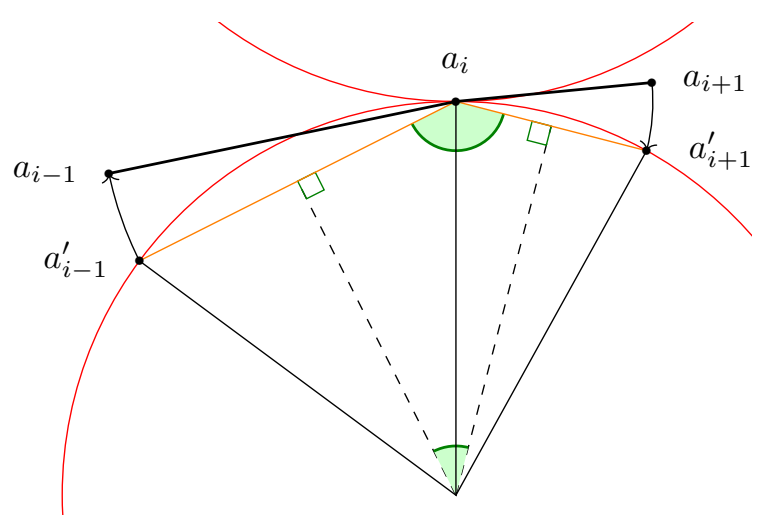

Figure 22: Thick, black: a polygonal line $\left[a_{i-1}, a_{i}, a_{i+1}\right]$ inscribed in a $\operatorname{par}(r)-$ regular curve (not depicted). The circles of par(r)-regularity are drawn in red. Thin, orange: a polygonal line $\left[a_{i-1}^{\prime}, a_{i}^{\prime}, a_{i+1}^{\prime}\right]$ inscribed in a circle with radius $r$ (the circle is the osculating circle that is on the side of the turn of the oriented polygonal line $\left.\left[a_{i-1}, a_{i}, a_{i+1}\right]\right)$. The polygonal lines have the same edge length sequence. 


\section{References}

[1] V.V. Alexandrov and Y.G. Reshetnyak. General Theory of Irregular Curves. Mathematics and its Applications. Springer Netherlands, 2012.

[2] A.I.B.T.U. Berlin, P. Schröder, J.M. Sullivan, and G.M. Ziegler. Discrete Differential Geometry. Oberwolfach Seminars. Birkhäuser Basel, 2008.

[3] Frédéric Chazal, David Cohen-Steiner, and André Lieutier. A sampling theory for compact sets in Euclidean space. Discrete 83 Computational Geometry, 41(3):461-479, Apr 2009.

[4] Herbert Federer. Curvature measures. Transactions of the American Mathematical Society, 93(3):418-491, 1959.

[5] Ari Gross and Longin Latecki. Digitizations preserving topological and differential geometric properties. Computer Vision and Image Understanding, 62(3):370 - 381, 1995.

[6] Rob Kusner and John Sullivan. On distortion and thickness of knots. Topology and Geometry in Polymer Science, 103, 031997.

[7] Jacques-Olivier Lachaud and Boris Thibert. Properties of gauss digitized shapes and digital surface integration. Journal of Mathematical Imaging and Vision, 54(2):162-180, Feb 2016.

[8] Longin Jan Latecki, Christopher Conrad, and Ari Gross. Preserving topology by a digitization process. Journal of Mathematical Imaging and Vision, 8(2):131-159, Mar 1998.

[9] Etienne Le Quentrec, Loïc Mazo, Etienne Baudrier, and Mohamed Tajine. Local turn-boundedness: a curvature control for a good digitization. In Michel Couprie, Jean Cousty, Yukiko Kenmochi, and Nabil Mustafa, editors, 21st IAPR International Conference on Discrete Geometry for Computer Imagery, Discrete Geometry for Computer Imagery, 21th IAPR International Conference, DGCI 2019, Paris, France, March 2019.

[10] Hans Meine, Ullrich Köthe, and Peer Stelldinger. A topological sampling theorem for robust boundary reconstruction and image segmentation. Discrete Applied Mathematics, 157(3):524-541, 2009. International Conference on Discrete Geometry for Computer Imagery.

[11] John W. Milnor. On the total curvature of knots. Annals of Mathematics, Second Series, 52:248-257, 1950.

[12] Phuc Ngo, Nicolas Passat, Yukiko Kenmochi, and Isabelle DebledRennesson. Geometric preservation of $2 \mathrm{D}$ digital objects under rigid motions. Journal of Mathematical Imaging and Vision, 61:204-223, 2019.

[13] T. Pavlidis. Algorithms for Graphics and Image Processing. Springer Berlin Heidelberg, 2012. 
[14] Jean Serra. Image Analysis and Mathematical Morphology. Academic Press, Inc., USA, 1983.

[15] Peer Stelldinger, Longin Jan Latecki, and Marcelo Siqueira. Topological equivalence between a $3 \mathrm{D}$ object and the reconstruction of its digital image. IEEE Trans. Pattern Anal. Mach. Intell., 29(1):126-140, January 2007.

[16] Peer Stelldinger and Kasim Terzic. Digitization of non-regular shapes in arbitrary dimensions. Image and Vision Computing, 26(10):1338-1346, 2008. 